\title{
Almost Radial Gauge
}

\author{
Andrzej Herdegen®
}

\begin{abstract}
An almost radial gauge $A^{\text {ar }}$ of the electromagnetic potential is constructed for which $x \cdot A^{\text {ar }}(x)$ vanishes arbitrarily fast in timelike directions. This potential is in the class introduced by Dirac with the purpose of forming gauge-invariant quantities in quantum electrodynamics. In the quantum case, the construction of smeared operators $A^{\mathrm{ar}}(K)$ is enabled by a natural extension of the free electromagnetic field algebra introduced earlier (represented in a Hilbert space). The space of possible smearing functions $K$ includes vector fields with the asymptotic spacetime behavior typical for scattered currents (the conservation condition in the whole spacetime need not be assumed). This construction is motivated by a possible application to the infrared problem in QED.
\end{abstract}

Mathematics Subject Classification. Primary 81V10; Secondary 81T05.

\section{Introduction}

The long-range nature of interaction entails the so-called infrared problems in electrodynamics, both classical and quantum [17]. Some of the quantum problems in this area are specific for this realm, but other reveal themselves already on the classical level. The identification of asymptotic charged fields and the scattering theory belong to the latter class.

The present author has long followed the idea that an appropriate choice of the electromagnetic gauge may relieve the scattering infrared problems. After preliminary results [11], it was shown recently that indeed the classical asymptotic problem for scattering of the Dirac field in electromagnetic timedependent field, typical for the complete theory, disappears in certain gauges. The main constituent feature of this class is sufficiently fast vanishing of the product $x \cdot A(x)$ for $x$ tending to timelike infinity (with an arbitrarily fixed origin $x=0$ in Minkowski space) [15]. The choice of a specific potential in this class has a large freedom in this classical context. However, we plan to extend 
our analysis to quantum electrodynamics, and in that case a judicious choice of our gauge is of fundamental importance.

The first gauge which probably comes to mind is the radial gauge $x$. $A^{\mathrm{rg}}(x)=0$, going also in the literature by the names Fock-Schwinger or relativistic Poincaré gauge $([9,24]$, see also [16]). In classical electrodynamics this gauge is well defined and may be obtained by integration of the electromagnetic field tensor. In quantum theory the latter property would be an advantage, as no indefinite metric space would be needed. However, we shall indicate in the following that the quantum version of this gauge is singular, which explains the need for a posteriori regularization in attempts to construct perturbative calculus with the use of this gauge [19].

It is the purpose of the present article to construct an 'almost radial gauge,' which may also be obtained from the electromagnetic field tensor alone, and for which $x \cdot A^{\text {ar }}(x)$ vanishes fast in timelike directions, but which at the same time admits quantization. Almost radial gauge turns out to be in the Dirac family of 'gauge-invariant gauges,' which in the standard formulation of QED may be constructed only after initial regularization and the use of the indefinite Gupta-Bleuler metric (see the discussion of this problem in [25], p. 191). We choose a different approach. For the construction of the quantum field, we use a natural extension of the algebra of the free field proposed earlier [12], which enables representation of infrared singular fields. This extension admits the construction of the almost radial gauge, and allows moreover its well-defined smearing with vector functions typically appearing in scattering.

As we shall see, the construction of the almost radial gauge involves integration of the electromagnetic field along radial $\mathbb{R}$-axes. This bears some similarity to the integration of the field along spacelike semi-axes, which is the principle of the construction of string-localized potentials introduced by Mund et al. [21]. We shall discuss similarities and differences of the two constructions.

The plan of the article is as follows. In Section 2, we summarize our notation and mathematical tools to be used in the article. Section 3 contains a brief summary of the quantization of the standard free field algebra, and its extension mentioned above. The classical radial gauge and its singularity in quantum case are discussed in Section 4. Section 5 contains our main results: construction and evaluation of properties of the almost radial gauge, both classical and quantum, along the lines announced above. In Section 6, we compare our construction with the string-localized potentials. Section 7 contains some final remarks. Proofs of some technical points are shifted to the Appendix.

\section{Preliminaries}

We list here our notation, conventions and preliminary formulas. More extensive summary of these questions may be found in [14].

We consider the flat spacetime with a fixed origin, thus described by the Minkowski vector space $M$ with the scalar product $x \cdot y$ with signature $(+,-,-,-)$. We choose physical units for which $\hbar, c$ and an arbitrarily chosen 
length scale are all equal to 1 . Then, in particular, $x \in M$ may be treated as dimensionless. We fix a unit timelike, future-pointing vector $t$ and for a Minkowski vector $x$ we write $x^{0}=x \cdot t$ and $\mathrm{x}=x-(x \cdot t) t$. The three-dimensional norm of $\mathbf{x}$ is denoted $|\mathbf{x}|$, and we also write $|x|=\left(\left|x^{0}\right|^{2}+|\mathbf{x}|^{2}\right)^{\frac{1}{2}}$. The tensor indices, which are often suppressed, are denoted by $a, b$ etc. The Minkowski volume measure element is denoted $\mathrm{d} x$. Our results do not depend on the choice of vector $t$.

\subsection{Homogeneous Functions on the Future Light Cone}

We shall write $l$ for any future-pointing lightlike (nonzero) vector and denote

$$
C_{+}=\left\{l \in M \mid l \cdot l=0, l^{0}>0\right\}, \quad C_{+}^{t}=\left\{l \in C_{+} \mid l^{0}=1\right\} .
$$

For $C^{1}$-functions $f(l)$ on $C_{+}$, the intrinsic differentiation operators

$$
L_{a b}=l_{a} \frac{\partial}{\partial l^{b}}-l_{b} \frac{\partial}{\partial l^{a}}
$$

are the generators of the Lorentz transformations $f(l) \mapsto f_{\Lambda}(l)=f\left(\Lambda^{-1} l\right)$, where $\Lambda$ is a Lorentz transformation of the Minkowski space. The derivative $\partial f / \partial l^{a}$ can only be given a unique meaning if one determines a particular $C^{1}$-extension of $f$ to a neighborhood of $C_{+}$. However, any two such extensions differ by a term $l^{2} g(l)$, with a $C^{1}$-function $g(l)$ in a neighborhood of $C_{+}$. Thus, on $C_{+}$the derivatives $\partial f(l)$ obtained from these two extensions differ by $2 l g(l)$. Therefore, if we define

$$
\partial_{a} f(l)=\left(l^{0}\right)^{-1} L_{0 a} f(l)
$$

in any chosen reference frame, then $\partial_{a} f(l)$ is uniquely defined as an equivalence class with respect to the addition of terms proportional to $l_{a}$. If the definition of $f(l)$ is the restriction of a function naturally defined in some neighborhood of $C_{+}$, then this function may also serve to define $\partial_{a} f(l)$.

For any measurable function $W(l)$ on $C_{+}$, homogeneous of degree -2 , the integral over the set of null directions defined by

$$
\int W(l) \mathrm{d}^{2} l=\int_{C_{+}^{t}} W(l) \mathrm{d} \Omega_{t}(l),
$$

where $\mathrm{d} \Omega_{t}(l)$ is the angle measure on the unit sphere, does not depend on the choice of the vector $t$. If $W$ is $C^{1}$, it follows that

$$
\int L_{a b} W(l) \mathrm{d}^{2} l=0 .
$$

Another closely related identity is the following. Let $V^{a}(l)$ be a $C^{1}$-vector function on $C_{+}$, such that $l \cdot V(l)=0$. Suppose $V(l)$ is extended to a neighborhood of the light cone with the preservation of these properties. Then in each Minkowski frame one has

$$
L_{0 a}\left(\frac{V^{a}}{l^{0}}\right)=\partial \cdot V(l),
$$


so the rhs of this equation is both frame- and extension (preserving orthogonality)-independent. Moreover, if in addition $V(l)$ is homogeneous of degree -1 , then

$$
\int \partial \cdot V(l) \mathrm{d}^{2} l=0 .
$$

If $f(l)$ is a scalar $C^{1}$-function, homogeneous of degree 0 , then the last identity and the earlier remarks on $\partial_{a} f(l)$ imply the 'integration by parts' identity

$$
\int f(l) \partial \cdot V(l) \mathrm{d}^{2} l=-\int V(l) \cdot \partial f(l) \mathrm{d}^{2} l .
$$

We end these remarks with the following definition. Let $F(l)$ be any continuous function, homogeneous of degree -1 . Then it is easily calculated that

$$
\lim _{\epsilon \searrow 0} \frac{1}{2 \pi} \int \delta\left(l \cdot l^{\prime}-\epsilon l^{0}{l^{\prime}}^{0}\right) F\left(l^{\prime}\right) \mathrm{d}^{2} l^{\prime}=F(l),
$$

independently of the frame in which the coordinates $l^{0} l^{\prime 0}$ are taken, where $\delta($. is the Dirac delta distribution. Therefore, we can interpret the lhs as the action of a distribution $\delta\left(l, l^{\prime}\right)$, homogeneous of degree -1 in each of the arguments:

$$
\int \delta\left(l, l^{\prime}\right) F\left(l^{\prime}\right) \mathrm{d}^{2} l^{\prime}=F(l) .
$$

\subsection{Fourier Transforms}

We use the following conventions for a function $f$ on $M$ and a function $g$ on $\mathbb{R} \times C_{+}$:

$$
\begin{aligned}
\hat{f}(p) & =\frac{1}{2 \pi} \int_{M} f(x) e^{i p \cdot x} \mathrm{~d} x, \\
\widetilde{g}(\omega, l) & =\frac{1}{2 \pi} \int_{\mathbb{R}} g(s, l) e^{i \omega s} \mathrm{~d} s .
\end{aligned}
$$

\subsection{Wave Equation}

Solutions of the homogeneous wave equation may be represented by

$$
A(x)=-\frac{1}{2 \pi} \int \dot{V}(x \cdot l, l) \mathrm{d}^{2} l,
$$

where $^{1} \dot{V}(s, l)$ (of any algebraic type) is a function homogeneous of degree -2 . This representation is related to the Fourier representation

$$
A(x)=\frac{1}{\pi} \int_{M} e^{-i x \cdot k} a(k) \operatorname{sgn}\left(k^{0}\right) \delta\left(k^{2}\right) \mathrm{d} k
$$

by

$$
\widetilde{\dot{V}}(\omega, l)=-\omega a(\omega l)
$$

\footnotetext{
${ }^{1}$ In what follows, the overdot will always denote the differentiation with respect to the real parameter $s$; for a choice of $V(s, l)$ see below.
} 
In physical contexts of interest the function $\dot{V}(s, l)$ is of class $C^{N}$, for some $N \in\{0,1, \ldots\} \cup\{\infty\}$, and for $l$ scaled $^{2}$ to $l^{0}=1$ and some $\varepsilon>0$ the following bounds hold:

$$
\left|L_{a_{1} b_{1}} \ldots L_{a_{k} b_{k}} \dot{V}(s, l)\right| \leq \frac{\text { const }}{(1+|s|)^{1+\varepsilon}}, \quad k=0,1, \ldots, N .
$$

With this condition, $A(x)$ has null asymptotes given by

$$
\lim _{R \rightarrow \infty} R A(x \pm R l)= \pm[V(x \cdot l, l)-V( \pm \infty, l)]
$$

where $V(s, l)$ is any primitive function of $\dot{V}(s, l)=\partial V(s, l) / \partial s$, determined up to an additive $s$-independent term. Independent of this choice is the difference

$$
\Delta V(l)=V(+\infty, l)-V(-\infty, l)=\int_{\mathbb{R}} \dot{V}(s, l) \mathrm{d} s .
$$

In what follows two spaces of functions $V(s, l)$ will appear naturally: one characterized by $V(+\infty, l)=0$, and another by $V(-\infty, l)=0$. In addition, we shall also find convenient to define another choice, denoted by

$$
\bar{V}(s, l)=\frac{1}{2} \int_{\mathbb{R}} \operatorname{sgn}(s-\tau) \dot{V}(\tau, l) \mathrm{d} \tau,
$$

for which

$$
\bar{V}( \pm \infty, l)= \pm \frac{1}{2} \Delta V(l)
$$

Beside the existence of null asymptotes, condition (5) entails (with the use of estimate (82)) the following decay of $A(x)$ in spacetime:

$$
\begin{aligned}
|A(x)| & \leq \text { const } \int \frac{\mathrm{d} \Omega_{t}(l)}{(1+|x \cdot l|)^{1+\varepsilon}}=\int_{-1}^{1} \frac{\text { const } \mathrm{d} u}{\left(1+|| x^{0}|+| \mathbf{x}|u|\right)^{1+\varepsilon}} \\
& \leq \frac{\text { const }}{1+\left|x^{0}\right|+|\mathbf{x}|}\left\{\theta\left(-x^{2}\right)+\frac{\theta\left(x^{2}\right)}{\left(1+\left|x^{0}\right|-|\mathbf{x}|\right)^{\varepsilon}}\right\} .
\end{aligned}
$$

We end this section by mentioning that the standard Pauli-Jordan function, a particular solution of the wave equation, has the representation

$$
D(x)=\frac{1}{2 \pi} \operatorname{sgn}\left(x^{0}\right) \delta\left(x^{2}\right)=-\frac{1}{8 \pi^{2}} \int \delta^{\prime}(x \cdot l) \mathrm{d}^{2} l .
$$

This may be shown by writing the rhs as

$$
-\frac{1}{8 \pi^{2}} \frac{\partial}{\partial x^{0}} \int \frac{\delta(x \cdot l)}{l^{0}} \mathrm{~d}^{2} l=-\frac{1}{4 \pi} \frac{\partial}{\partial x^{0}} \int_{-1}^{1} \delta\left(x^{0}+|\mathbf{x}| u\right) \mathrm{d} u=-\frac{1}{4 \pi} \frac{\partial}{\partial x^{0}} \frac{\theta\left(-x^{2}\right)}{|\mathbf{x}|},
$$

which gives the standard form.

\footnotetext{
${ }^{2}$ This scaling is to be understood in all further estimates, to appear below, containing $s$ or $l$.
} 


\subsection{Free Electromagnetic Field}

A Lorenz potential $A$ of a free electromagnetic field $F$ has the representation (4), where $\dot{V}(s, l)$ is a vector function satisfying the condition

$$
l \cdot \dot{V}(s, l)=0 \text {. }
$$

We assume that the condition (5) with $N \geq 1$ is satisfied, so $\Delta V(l)$ exists and satisfies $l \cdot \Delta V(l)=0$. Moreover, we demand in addition that

$$
L_{[a b} \Delta V_{c]}(l)=0,
$$

which under the orthogonality condition is equivalent to the existence of a homogeneous of degree 0 function $\Phi(l)$, such that

$$
\Delta V_{a}(l)=-\partial_{a} \Phi(l),
$$

equality in the sense described in Section 2.1. Then $A$ is of the type of radiation potentials of fields created in scattering processes. The corresponding electromagnetic field is then given by

$$
F_{a b}(x)=-\frac{1}{2 \pi} \int\left[l_{a} \ddot{V}_{b}(x \cdot l, l)-l_{b} \ddot{V}_{a}(x \cdot l, l)\right] \mathrm{d}^{2} l .
$$

The gauge freedom within the space of Lorenz gauges is represented in terms of $\dot{V}(s, l)$ by the transformations

$$
\dot{V}(s, l) \mapsto \dot{V}(s, l)+l \dot{\alpha}(s, l),
$$

with $\alpha(s, l)$ homogeneous of degree -2 . The spacelike asymptotic behavior of $A$ (and $F$ ) depends on $\Delta V(l)$ and is given, independently of $x$, by

$$
\lim _{R \rightarrow \infty} R A(x+R y)=-\frac{1}{2 \pi} \int \Delta V(l) \delta(y \cdot l) \mathrm{d}^{2} l, \quad y^{2}<0 .
$$

Fields with $\Delta V(l) \neq 0$ are usually termed as infrared singular; their spacelike tail falls off as that of the Coulomb field of the electric charge.

For future use, we note the following representation:

$$
x \cdot A(x)=\frac{1}{2 \pi} \int(\partial \cdot V)(x \cdot l, l) \mathrm{d}^{2} l ;
$$

here and in what follows we denote

$$
\left(\partial_{a} V\right)(x \cdot l, l)=\left.\frac{\partial}{\partial l^{a}} V(s, l)\right|_{s=x \cdot l}
$$

(and similarly for $L_{a b}$ and other functions in place of $V$ ). Representation (15) follows immediately from the relation

$$
\frac{\partial}{\partial l} \cdot V(x \cdot l, l)=x \cdot \dot{V}(x \cdot l, l)+(\partial \cdot V)(x \cdot l, l)
$$

by the use of identity (1).

Finally, it is important for what comes to relate representation (4) to the construction of the radiation potential of a conserved vector current $J(x)$,

$$
\partial \cdot J(x)=0 .
$$


Let $J(x)$ be such current, for which $\dot{V}(s, l)$, with

$$
V(s, l)=\int J(x) \delta(s-x \cdot l) \mathrm{d} x,
$$

satisfies conditions (5). Then the radiation potential of this current has two equivalent forms

$$
A[J](x)=4 \pi \int_{M} D(x-y) J(y) \mathrm{d} y=-\frac{1}{2 \pi} \int \dot{V}(x \cdot l, l) \mathrm{d}^{2} l,
$$

which is shown with the use of representation (9). We note that orthogonality (10) is then automatic, but in general

$$
l \cdot V(s, l)=Q
$$

$Q$ being the charge of the current.

\section{Free Quantum Field}

Quantization of the local free electromagnetic field may be described in terms of the algebra of electromagnetic potential elements $A(J)$ satisfying the relations

$$
\left[A\left(J_{1}\right), A\left(J_{2}\right)\right]=i 4 \pi \int J_{1}(x) D(x-y) J_{2}(y) \mathrm{d} x \mathrm{~d} y=i\left\{J_{1}, J_{2}\right\}
$$

where $J_{i}$ are smooth, compactly supported conserved test currents (as discussed in [12] and [13]). After exponentiation to the Weyl form, this defines a net of $C^{*}$-algebras satisfying Einstein causality in the form of commutation for spacelike separated supports of $J_{i} \cdot{ }^{3}$ This formulation is close to the discussion given in [23]. In more standard formulation one treats the free electromagnetic field as an operator-valued distribution on the space of antisymmetric, smooth, compactly supported functions $\varphi^{a b}(x)$. Then the elements

$$
F(\varphi)=A(J), \quad \text { where } \quad J^{a}=2 \partial_{b} \varphi^{a b},
$$

generate the standard net of electromagnetic field, where now $F(\varphi)$ is considered as an element of the local algebra of any open set containing $\operatorname{supp} \varphi$. However, as each conserved, compactly supported current $J^{a}$ has a representation (20) with a compactly supported ${ }^{4} \varphi^{a b}$, the nets have the same elements, with some differences in the assignment to spacetime sets. Moreover, two important properties bind the two nets:

\footnotetext{
${ }^{3}$ More recently, description in terms of 'intrinsic potential' $A(J)$ has been used for an axiomatic construction of a general local electromagnetic algebra in [6]. In that case, according to this analysis, the commutators for spacelike separated, but topologically nontrivial regions need not vanish, but are in the center of the algebra.

${ }^{4}$ This more specific Poincaré lemma for compact supports follows from its standard form and the properties of cohomology of spheres, see e.g., [18], Lemma 17.27. Also, the proof in that reference easily implies that for supp $J$ contained in an open, star-shaped set, there exists $\varphi$ with support contained in the same set. See also [6].
} 
(i) the nets are local with respect to each other, i.e.,

$$
[F(\varphi), A(J)]=0
$$

for spacelike separated supports of $\varphi$ and $J$;

(ii) for each open star-shaped set the two local algebras assigned to this set are equal.

The first property is obvious from (19) and (20), and for the second see footnote 4 .

The symplectic form on the rhs of (19) may be expressed in terms of functions $V(s, l)$ defined by (16). Substituting in this form representation (9), one obtains ${ }^{5}$

$$
\begin{aligned}
\left\{V_{1}, V_{2}\right\} & =\frac{1}{4 \pi} \int\left[\dot{V}_{1}(s, l) \cdot V_{2}(s, l)-V_{1}(s, l) \cdot \dot{V}_{2}(s, l)\right] \mathrm{d} s \mathrm{~d}^{2} l \\
& =i \int \widetilde{\dot{V}}_{1}(\omega, l) \cdot \tilde{\dot{V}}_{2}(\omega, l) \frac{\mathrm{d} \omega}{\omega} \mathrm{d}^{2} l,
\end{aligned}
$$

where for the Fourier-transformed version we used (3). If one writes symbolically $A\left(J_{i}\right)=\int_{M} A(x) J_{i}(x) \mathrm{d} x$, with $A(x)$ represented by (4) with quantum function $V^{\mathrm{q}}(s, l)$, then

$$
A\left(J_{i}\right)=\left\{V_{i}, V^{\mathrm{q}}\right\} .
$$

The quantization condition (19) takes now the form

$$
\left[\left\{V_{1}, V^{\mathrm{q}}\right\},\left\{V_{2}, V^{\mathrm{q}}\right\}\right]=i\left\{V_{1}, V_{2}\right\} .
$$

This condition is now extended to the space of all smooth, compactly supported functions $V(s, l)$, satisfying orthogonality ${ }^{6}$

$$
l \cdot V(s, l)=0,
$$

which for $V(s, l)$ obtained from $J$ reflects the fact that the current is chargeless. The form (21) is nondegenerate on the space of equivalence classes of such functions with respect to gauge transformations (13). This may be also interpreted as follows: the symplectic structure is defined and nondegenerate on the space of infrared regular classical fields (12). This leads to the unique Weyl algebra satisfying the corresponding exponentiated condition on this space (see [4], Section 5.2.2.2). Consider the scalar product on the space of equivalence classes

$$
\left(\tilde{\dot{V}}_{1}, \tilde{\dot{V}}_{2}\right)=\iint_{\mathbb{R}_{+}}\left[-\overline{\widetilde{\dot{V}}_{1}(\omega, l)} \cdot \widetilde{\dot{V}}_{2}(\omega, l)\right] \frac{\mathrm{d} \omega}{\omega} \mathrm{d}^{2} l,
$$

and the Hilbert space $\mathcal{H}$ obtained by completion. The standard construction leads to the representation of the algebra on the Fock space based on $\mathcal{H}$. When restricted to the exponentiated elements $F(\varphi)$, this is the standard irreducible vacuum representation of the local net.

\footnotetext{
${ }^{5}$ By a slight abuse of notation, we use here the same symbol as in (19).

${ }^{6}$ This formulation of the quantization is in the spirit of 'asymptotic quantization' discussed by Bramson [3] and later by Ashtekar [1]).
} 
In interacting theory one needs to form elements $A(K)$, where the vector function $K$ need not be conserved, and secondly, need not be compactly supported. The first problem (non-conservation of $K$ ) is dealt by choosing $A$ in some special gauge, and in local formulation of QED this leads to the GuptaBleuler indefinite metric. The second problem (non-compactness) is avoided in usual formulations by the introduction of a switching function $g(x)$ and the subsequent performance of the 'adiabatic limit' $g \rightarrow 1$. This program of the solution of infrared problems is far from complete. ${ }^{7}$ Here we want to propose a construction avoiding both problems, with the use of a natural extension of the above algebra to the free infrared singular fields, put forward in [12]. We briefly sketch the construction of this extension.

The symplectic structure (21) has a natural extension to a wider space. It is easy to see that the form remains well defined (absolutely integrable) and gauge invariant for general functions $V_{i}$ subject to conditions (5) with $N=\infty$, (11) and (24). However, for this structure to be again consistently formulated on the space of all classical fields (12) (now also those infrared singular when $\left.\Delta V_{i}(l) \neq 0\right)$, we have to make the choice of $V(s, l)$ for given $\dot{V}(s, l)$ unique. We make this choice guided by the following physical motivation (as discussed in [13]). Free fields appear in realistic physical theories as incoming or outgoing fields in scattering setting. We want to admit the possibility of testing these fields with currents infinitely extended in timelike directions. ${ }^{8}$ Formula (16) tells us that for $V(+\infty, l)(V(-\infty, l))$ to be different from zero, the test current $J$ must have a sufficiently slow (in fact, of $|x|^{-3}$ type) decay in the future (past), respectively. Therefore, it seems reasonable to adopt the rule that

$$
\begin{array}{ll}
V(+\infty, l)=0 & \text { for 'in' case, } \\
V(-\infty, l)=0 & \text { for 'out' case, }
\end{array}
$$

consistently with the spacetime regions where the fields are tested: 'in' in the past, and 'out' in the future; the same rule is adopted for the quantum variables $V^{\mathrm{q}}(s, l)$. Let us mention that all such extended currents are chargefree (see (18)) and that the symplectic form $\left\{V_{1}, V_{2}\right\}$ for such currents may be represented in the form

$$
\left\{J_{1}, J_{2}\right\}=\frac{1}{2} \int_{M}\left(A\left[J_{1}\right] \cdot J_{2}-A\left[J_{2}\right] \cdot J_{1}\right)(x) \mathrm{d} x,
$$

where $A\left[J_{i}\right](x)$ are radiation fields (17). For compactly supported $J_{i}$ this agrees with the definition in (19); however, the latter is not absolutely integrable in general.

Relations (23), considered on the space of functions $V(s, l)$ satisfying (5) with $N=\infty,(11),(24)$ and (26), define (after exponentiation) a $C^{*}$ algebra, an extension of the standard local algebra. Algebras for 'in' and 'out' cases are canonically isomorphic. For $J_{i}$ with supports spacelike separated the usual commutation rule is fulfilled. Also, the form (27) shows that the timelike commutativity characteristic for massless fields extends as well: if $\operatorname{supp} J_{1}$ is

\footnotetext{
${ }^{7}$ But see a recent contribution to this program [8].

${ }^{8}$ Extensive discussion of such test currents may be found in [13]; see also what follows.
} 
timelike separated from supp $J_{2}$, then the two fields commute, irrespective of the size of the supports.

The Poincaré group transformations $(z, \Lambda)$ are represented by the automorphisms $\alpha_{z, \Lambda}$ acting on the algebra by

$$
\alpha_{z, \Lambda}[A(J)]=A\left(T_{z, \Lambda} J\right),
$$

where $T_{z, \Lambda}$ is the natural representation of the Poincaré group on tensor functions. Expressed in terms of notation (22), this reads

$$
\alpha_{z, \Lambda}\left[\left\{V, V^{\mathrm{q}}\right\}\right]=\left\{T_{z, \Lambda} V, V^{\mathrm{q}}\right\}
$$

where

$$
\left[T_{z, \Lambda} V\right]_{a}(s, l)=\Lambda_{a}{ }^{b} V_{b}\left(s-z \cdot l, \Lambda^{-1} l\right) .
$$

Translationally covariant, positive energy, irreducible representations of the extended algebra have been constructed in [12] and [13]. Also, there exist among them those for which rotations are implemented, although not Lorentz boosts. In these representations there are vector states with arbitrarily small energy content, but no vacuum vector state exists.

The integral in the Fourier-transformed version of the symplectic form (21) is now in the principal value sense. Note also that $\widetilde{\dot{V}}(0, l)=(2 \pi)^{-1} \Delta V(l)$. Therefore, elements $\left\{V_{1}, V^{\mathrm{q}}\right\}$ with $\Delta V_{1}(l) \neq 0$, describing infrared singular fields, have no representation in the vacuum representation of the local algebra, which is based on the scalar product (25). We shall see in Section 5 that our larger arena enables the construction of elements $A^{\mathrm{ar}}(K)$ for appropriately extended functions $K$ and almost radial gauge $A^{\text {ar }}$ to be defined there.

For later comparison, we write the symbolic distributional formula equivalent to (23) (for test functions $V_{i}$ vanishing at infinity):

$$
\left[\dot{V}_{a}^{\mathrm{q}}(s, l), \dot{V}_{b}^{\mathrm{q}}\left(s^{\prime}, l^{\prime}\right)\right]=-i g_{a b} \frac{2 \pi}{l^{0} l^{\prime^{0}}} \delta\left(l, l^{\prime}\right) \delta^{\prime}\left(\frac{s}{l^{0}}-\frac{s^{\prime}}{l^{\prime 0}}\right) .
$$

The rhs has the correct homogeneity and is independent of the choice of the vector $t$. The metric tensor on the rhs could suggest indefinite metric, but this is not the case: this relation is smeared with (equivalence classes with respect to (13) of) functions $V_{i}(s, l)$ orthogonal to $l$. In local formulation of interacting theory one needs to smear $A(x)$ with non-conserved test vector fields, and then the usual Gupta-Bleuler formulation is needed.

Finally, let us note for future use that if both $V_{i}(s, l)$ satisfy one and the same of the conditions (26), then

$$
\left\{V_{1}, V_{2}\right\}=\left\{\bar{V}_{1}, \bar{V}_{2}\right\},
$$

where $\bar{V}_{i}(s, l)$ are formed by the convention $(7)$.

\section{Radial Gauge}

As announced in the Introduction, we are now looking for a suitable quantum gauge, in which $x \cdot A(x)$ vanishes (sufficiently fast) in timelike directions. We start by testing the radial gauge for that purpose, for a classical field first. 
If $F_{a b}(x)=\partial_{a} A_{b}(x)-\partial_{b} A_{a}(x)$, with $A$ a $C^{1}$-function, then without any further conditions the following identity holds:

$$
x^{a} F_{a b}(x)=(x \cdot \partial+1) A_{b}(x)-\partial_{b}(x \cdot A(x)),
$$

so for $\lambda \in \mathbb{R}$ we have

$$
\lambda x^{a} F_{a b}(\lambda x)=\frac{\partial}{\partial \lambda}\left[\lambda A_{b}(\lambda x)\right]-\partial_{b}(x \cdot A(\lambda x)) .
$$

Integrating with respect to $\lambda$ on $[0,1]$, one finds

$$
\begin{aligned}
\int_{0}^{1} \lambda x^{a} F_{a b}(\lambda x) \mathrm{d} \lambda & =A_{b}(x)-\partial_{b} S^{\mathrm{rg}}(x) \equiv A_{b}^{\mathrm{rg}}(x), \\
S^{\mathrm{rg}}(x) & =\int_{0}^{1} x \cdot A(\lambda x) \mathrm{d} \lambda,
\end{aligned}
$$

which for any electromagnetic field $F_{a b}$ defines the radial gauge with the property

$$
x \cdot A^{\mathrm{rg}}(x)=0 .
$$

Potential (33) is the unique regular solution of the condition (34). Indeed, any addition to $A_{a}^{\mathrm{rg}}(x)$ of a gauge term $\partial_{a} G(x)$ respecting (34) must result from a homogeneous function $G(x)$, which has to be singular (not continuous) at the origin, if it is not constant.

The lhs of (33) shows immediately that for $F$ - a free Maxwell field, the radial gauge is a Lorenz gauge. Let $A(x)$ be a Lorenz gauge as specified in Section 2.4. Then one finds

$$
A^{\mathrm{rg}}(x)=-\frac{1}{2 \pi} \int \dot{V}^{\mathrm{rg}}(x \cdot l, l) \mathrm{d}^{2} l,
$$

with

$$
\dot{V}_{b}^{\mathrm{rg}}(s, l)=\dot{V}_{b}(s, l)+l_{b} \frac{\partial \cdot V(s, l)-\partial \cdot V(0, l)}{s} .
$$

This is shown by noting that by (15) we have

SO

$$
x \cdot A(\lambda x)=\frac{1}{2 \pi \lambda} \int(\partial \cdot V)(\lambda x \cdot l, l) \mathrm{d}^{2} l,
$$

$$
S^{\mathrm{rg}}(x)=\frac{1}{2 \pi} \int_{0}^{1}\left(\int(\partial \cdot V)(\lambda x \cdot l, l) \mathrm{d}^{2} l\right) \frac{\mathrm{d} \lambda}{\lambda}
$$

and

$$
\begin{aligned}
\partial_{b} S^{\mathrm{rg}}(x) & =\frac{1}{2 \pi} \int_{0}^{1} \int l_{b}(\partial \cdot \dot{V})(\lambda x \cdot l, l) \mathrm{d}^{2} l \mathrm{~d} \lambda \\
& =\frac{1}{2 \pi} \int l_{b} \frac{(\partial \cdot V)(x \cdot l, l)-(\partial \cdot V)(0, l)}{x \cdot l} \mathrm{~d}^{2} l
\end{aligned}
$$

(note that $S^{\mathrm{rg}}(x)$ is well defined, since $\left.\int(\partial \cdot V)(0, l) \mathrm{d}^{2} l=0\right)$.

For a classical field, with sufficiently regular $V(s, l)$, the radial gauge function (36) is regular and the representation (35) is well defined (e.g., for a smooth $V(s, l)$ both $V^{\mathrm{rg}}(s, l)$ and $A^{\mathrm{rg}}(x)$ are smooth), although with marked 
differences as compared to $A$. In contrast to $\dot{V}(s, l)$, which satisfies $(5), \dot{V}^{\mathrm{rg}}(s, l)$ decays only as $s^{-1}$ for large $|s|$. This has two consequences: first, there is no null asymptotics of the form (6) for $A^{\mathrm{rg}}(x)$; and second, the estimate (8) is replaced by a slower decay

$$
\left|A^{\mathrm{rg}}(x)\right| \leq \mathrm{const} \int \frac{\mathrm{d} \Omega(l)}{1+|x \cdot l|} \leq \frac{\mathrm{const}}{1+\left|x^{0}\right|+|\mathbf{x}|} \log (2+|\mathbf{x}|),
$$

which is easily obtained with the use of estimate (82).

However, for the quantum version it follows immediately from the juxtaposition of Formulas (36) and (30) that $V^{\mathrm{rg}}(s, l)$, and consequently also $A^{\mathrm{rg}}(x)$, are not properly defined, due to the appearance of a distributional quantity at a point: $\partial \cdot V(0, l)$. This, in our view, is the fundamental reason for the reported difficulties and a need for a posteriori regularization in attempts to establish Feynman rules in the radial gauge (see [19] and references therein).

\section{Almost Radial Gauge}

The source of trouble in the radial gauge is the term $\partial \cdot V(0, l)$ in the function $\dot{V}^{\mathrm{rg}}(s, l)$. Our plan is to modify the definition (33) so as to eliminate this term. This will only be possible with some smearing of the central, reference point. The result of this program is the construction of an almost radial gauge potential $A_{b}^{\text {ar }}(x)$ in Formulas (49), (50), (51) and (72) below.

The starting point for the construction is the following variant of the identity (32)

$$
(\xi+1) \overrightarrow{a x}^{a} F_{a b}(x+\xi \overrightarrow{a x})=\frac{\partial}{\partial \xi}\left[(\xi+1) A_{b}(x+\xi \overrightarrow{a x})\right]-\partial_{b}^{x}(\overrightarrow{a x} \cdot A(x+\xi \overrightarrow{a x})),
$$

where $\xi$ is a real parameter, and to abbreviate notation we introduced a usual affine space symbol

$$
\overrightarrow{a x}=x-a .
$$

To obtain this identity, we shift the argument of $A$ and $F$ in (32) by vector $a$, and write it with $x$ replaced by $y$ :

$$
\lambda y^{a} F_{a b}(a+\lambda y)=\frac{\partial}{\partial \lambda}\left[\lambda A_{b}(a+\lambda y)\right]-\frac{\partial}{\partial y^{b}}(y \cdot A(a+\lambda y)) .
$$

Now the substitutions $y=\overrightarrow{a x}$ and $\lambda=\xi+1$ give the result.

\subsection{Potential of Fast Decay}

We start discussion with the case of $A_{b}(x)$ a $C^{1}$-function of fast decay, together with its derivative. For such function one could think of integrating identity (37) with respect to $\xi$ on $[0, \infty)$, which would correspond to the $\lambda$-integration of $(32)$ on $[1, \infty)$. However, for $F$-a free electromagnetic field, which will be our object of study later, this would not be convergent for spacelike $\overrightarrow{a x}$. In that case, one has to exploit the oddness (in the spacetime) of its spacelike tail (and evenness of the tail of $A$, see (14)) and define integrals as appropriate 
limits. Anticipating this, we multiply $(37)$ by $\left(-\frac{1}{2}\right) \operatorname{sgn}(\xi)$ and integrate with respect to $\xi$ on $\mathbb{R}$, which results in

$$
\begin{gathered}
-\frac{1}{2} \int_{\mathbb{R}} \operatorname{sgn}(\xi)(\xi+1) \overrightarrow{a x}^{a} F_{a b}(x+\xi \overrightarrow{a x}) \mathrm{d} \xi=A_{b}(x)-\partial_{b}^{x} S(a, x), \\
S(a, x)=-\frac{1}{2} \int_{\mathbb{R}} \overrightarrow{a x} \cdot A(x+\xi \overrightarrow{a x}) \operatorname{sgn}(\xi) \mathrm{d} \xi \\
=-\frac{1}{2} \int_{\mathbb{R}} \overrightarrow{a x} \cdot A(a+\lambda \overrightarrow{a x}) \operatorname{sgn}(\lambda-1) \mathrm{d} \lambda .
\end{gathered}
$$

Using a technique similar to that applied in Appendix B to analyze function $r^{a}(x, z)$ defined below, it is easy to show that $S(a, x)$ is regular outside $x=a$, bounded by a constant, while $\left|\partial^{x} S(a, x)\right| \leq \operatorname{const}(1+|a|)|x-a|^{-1}$ (for that, use the second formula in (39)). In order to remove the singularity, we smear (38) with a real Schwartz function $\rho(a)$ on Minkowski space, such that

$$
\int_{M} \rho(a) \mathrm{d} a=1, \quad \int_{M} \rho(a) a^{\alpha} \mathrm{d} a=0 \quad \text { for } \quad 1 \leq|\alpha| \leq n,
$$

for some arbitrarily chosen (large) $n \in \mathbb{N} \cup\{\infty\}$. The smearing of $\partial^{x} S(a, x)$ may be pulled under the differential sign, and we obtain

$$
\begin{aligned}
\int_{M} S(a, x) \rho(a) \mathrm{d} a & =\frac{1}{2} \int_{M} A(y) \cdot \overrightarrow{y x} \int_{\mathbb{R}} \rho\left(x+\xi^{-1} \overrightarrow{y x}\right)|\xi|^{-5} \mathrm{~d} \xi \mathrm{d} y \\
& =\frac{1}{2} \int_{M} A(y) \cdot \overrightarrow{y x} \int_{\mathbb{R}} \rho(x+u \overrightarrow{y x})|u|^{3} \mathrm{~d} u \mathrm{~d} y .
\end{aligned}
$$

In the first step above we substituted $S(a, x)$ (39) and changed integration variable $a$ to $y=x+\xi(x-a)$. In the second step we substituted $\xi=u^{-1}$.

We now introduce the vector function

$$
r^{a}(x, z)=\frac{1}{2} z^{a} \int_{\mathbb{R}} \rho(x+u z)|u|^{3} \mathrm{~d} u,
$$

which together with its derivatives with respect to $x$ is estimated as in (83) and satisfies the distributional equation (see Appendix B)

$$
\partial^{z} \cdot r(x, z)=\delta(z)
$$

and define the almost radial gauge by

$$
\begin{aligned}
A_{b}^{\mathrm{ar}}(x) & =-\frac{1}{2} \int_{M} \rho(a) \int_{\mathbb{R}} \operatorname{sgn}(\xi)(\xi+1) \overrightarrow{a x}^{a} F_{a b}(x+\xi \overrightarrow{a x}) \mathrm{d} \xi \mathrm{d} a \\
& =A_{b}(x)-\partial_{b} S^{\mathrm{ar}}(x), \\
S^{\operatorname{ar}}(x) & =\int_{M} r(x, x-y) \cdot A(y) \mathrm{d} y=\int_{M} r(x, z) \cdot A(x-z) \mathrm{d} z .
\end{aligned}
$$

Formulas (42), (44) and (45) together show that our almost radial gauge is in the class of generalized 'gauge-invariant gauges' first postulated by Dirac [7] in an attempt to obtain 'physical,' out of local potentials (see also [25]). The generalization consists in nontrivial dependence of $r^{a}(x, x-y)$ on the 
first argument. Potential $A^{\text {ar }}(x)$ is a continuous function, decaying as $|x|^{-1}$ for large $|x|$, thus

$$
\left|A^{\mathrm{ar}}(x)\right| \leq \frac{\mathrm{const}}{1+|x|} .
$$

To estimate the radial component of $A^{\text {ar }}$, assume for simplicity that the support of $\rho(a)$ is contained in a small ball $|a| \leq \delta$, and note that $\overrightarrow{a x}^{a} x^{b} F_{a b}=$ $\overrightarrow{a x}^{a} a^{b} F_{a b}$. Then the contraction of (43) with $x$ gives

$$
\left|x \cdot A^{\operatorname{ar}}(x)\right| \leq \delta \frac{\text { const }}{1+|x|} .
$$

In the present case of a fast decaying function $A$, the 'almost radial' property has only a global aspect, appearance of $\delta$ on the rhs. It is the case of free field discussed below, where this term has a fuller justification.

We note that a strict Dirac gauge with $r(0, x-y)$ replacing $r(x, x-y)$ in (45) is also possible, but it does not have the almost radial property.

\subsection{Free Classical Field}

We now assume that $F_{a b}(x)$ is a free classical field obtained from the potential (4), where $\dot{V}(s, l)$ satisfies all restrictions formulated there. The definition (45) may not be directly applied in this case, but we shall find that an extension of this definition may be obtained as a limit of a regularized expression. We admit infrared singular fields, thus $\Delta V(l)$ may be different from zero. For the sake of the construction of almost radial gauge at the classical level, it will prove convenient to use the convention $\bar{V}(s, l)(7)$. The choice (26) will be restored at the stage of quantization.

The following auxiliary functions will be needed:

$$
\begin{aligned}
\sigma(s, l) & =\frac{1}{2} \int_{M} \operatorname{sgn}(s-a \cdot l) \rho(a) \mathrm{d} a \\
\zeta(s, l) & =\int_{M} \frac{\rho(a)}{s-a \cdot l} \mathrm{~d} a=\int_{\mathbb{R}} \frac{\dot{\sigma}(\tau, l)}{s-\tau} \mathrm{d} \tau, \\
\eta(s, l) & =\int_{M} \frac{\rho(a) a}{s-a \cdot l} \mathrm{~d} a=-\int_{\mathbb{R}} \frac{\partial \sigma(\tau, l)}{s-\tau} \mathrm{d} \tau
\end{aligned}
$$

(we assume $\sigma(s, l)$ is defined by the above formula also in a neighborhood of the light cone, so $\partial \sigma(s, l)$ is also unique in this case). Properties of these functions are discussed in Appendix C. Let $\chi(x)$ be a Schwartz function, such that

$$
\chi(-x)=\chi(x), \quad \chi(0)=1,
$$

and for $\delta>0$ denote (cf. (45))

$$
S_{\delta}^{\operatorname{ar}}(x)=\int_{M} \chi(\delta z) r(x, z) \cdot A(x-z) \mathrm{d} z,
$$

with $A(x)$ as described above. Then the following limit exists:

$$
S^{\operatorname{ar}}(x)=\lim _{\delta \searrow 0} S_{\delta}^{\operatorname{ar}}(x)=\frac{1}{2 \pi} \int[\eta(x \cdot l, l)-\zeta(x \cdot l, l) x] \cdot \bar{V}(x \cdot l, l) \mathrm{d}^{2} l .
$$


We give the proof of this fact in Appendix D. Gradient of this function is easily obtained

$\partial_{b} S^{\operatorname{ar}}(x)=\left.\frac{1}{2 \pi} \int\left\{l_{b} \partial_{s}[(\eta(s, l)-\zeta(s, l) x) \cdot \bar{V}(s, l)]-\zeta(s, l) \bar{V}_{b}(s, l)\right\}\right|_{s=x \cdot l} \mathrm{~d}^{2} l$.

We use an identity of the form (15) to transform

$$
-\left.\frac{1}{2 \pi} x^{a} \int l_{b} \partial_{s}\left[\bar{V}_{a}(s, l) \zeta(s, l)\right]\right|_{s=x \cdot l} \mathrm{~d}^{2} l=\left.\frac{1}{2 \pi} \int \partial_{a}\left[l_{b} \bar{V}^{a}(s, l) \zeta(s, l)\right]\right|_{s=x \cdot l} \mathrm{~d}^{2} l .
$$

Taking into account that $\partial_{a}\left[l_{b} \bar{V}^{a} \zeta\right]=\bar{V}_{b} \zeta+l_{b} \partial \cdot[\bar{V} \zeta]$, we find

$$
\partial_{b} S^{\mathrm{ar}}(x)=\left.\frac{1}{2 \pi} \int l_{b}\left\{\partial_{s}[\eta(s, l) \cdot \bar{V}(s, l)]+\partial \cdot[\zeta(s, l) \bar{V}(s, l)]\right\}\right|_{s=x \cdot l} \mathrm{~d}^{2} l .
$$

We can now extend the definition of the almost radial gauge to the free field:

$$
A_{b}^{\mathrm{ar}}(x)=A_{b}(x)-\partial_{b} S^{\mathrm{ar}}(x)=-\frac{1}{2 \pi} \int \dot{V}_{b}^{\mathrm{ar}}(x \cdot l, l) \mathrm{d}^{2} l
$$

where

$$
\begin{aligned}
\dot{V}^{\mathrm{ar}}(s, l) & =\dot{\bar{V}}(s, l)+l\left\{\partial_{s}[\eta(s, l) \cdot \bar{V}(s, l)]+\partial \cdot[\zeta(s, l) \bar{V}(s, l)]\right\} \\
& =\dot{\bar{V}}(s, l)+l\{\eta(s, l) \cdot \dot{\bar{V}}(s, l)+\zeta(s, l) \partial \cdot \bar{V}(s, l)\}
\end{aligned}
$$

the second form (51) obtained with the use of identity (84). Our almost radial gauge is a functional of the smearing function $\rho(40)$, which serves to define functions $\zeta$ and $\eta$ (46). Note that putting formally $\rho(a)=\delta(a)$ (which, precisely, is not allowed), one has $\zeta(s, l)=s^{-1}, \eta(s, l)=0$ and (51) reproduces Formula (36) with the term $(\partial \cdot V)(0, l)$ omitted (which, again, would result in a singularity).

The almost radial gauge is again a Lorenz gauge satisfying the wave equation. It is also gauge invariant with respect to gauge transformations

$$
A_{b}(x) \rightarrow A_{b}(x)+\partial_{b} \Lambda(x), \quad \Lambda(x)=-\frac{1}{2 \pi} \int \bar{\alpha}(x \cdot l, l) \mathrm{d}^{2} l,
$$

which correspond to the replacement $\bar{V}_{b}(s, l) \rightarrow \bar{V}_{b}(s, l)+l_{b} \bar{\alpha}(s, l)$. This replacement leaves $\dot{V}_{b}^{\text {ar }}(s, l)$ unchanged. To show this, we put $l \bar{\alpha}(s, l)$ in place of $\bar{V}(s, l)$ in $(51)$. Recall that to calculate $\partial \cdot[l \bar{\alpha}(s, l)]$ one has to extend $l \bar{\alpha}(s, l)$ to the neighborhood of the light cone respecting orthogonality to $l$, e.g., by

$$
l_{a} \bar{\alpha}(s, l) \rightarrow\left[l_{a} \bar{\alpha}(s, l)\right]_{\mathrm{ext}}=\left[l_{a}-\left(l^{0}\right)^{-1} l^{2} \delta_{a}^{0}\right] \bar{\alpha}(s, l),
$$

and then on the light cone $\partial \cdot[l \bar{\alpha}(s, l)]_{\text {ext }}=(l \cdot \partial+2) \bar{\alpha}(s, l)$. In this way the change in $(51)$ becomes

$$
l \zeta(s, l)\left(s \partial_{s}+l \cdot \partial+2\right) \bar{\alpha}(s, l)=0,
$$

where we used identity (85) and the homogeneity of degree -2 of $\bar{\alpha}(s, l)$.

Similarly as in the radial gauge case, $\dot{V}^{\text {ar }}(s, l)$ decays only as $1 / s$ for $|s| \rightarrow \infty$, so $A^{\text {ar }}(x)$ has no null asymptotes and decays as

$$
\left|A^{\operatorname{ar}}(x)\right| \leq \frac{\text { const }}{1+\left|x^{0}\right|+|\mathbf{x}|} \log (2+|\mathbf{x}|) .
$$


On the other hand, using identity (85) we find

$$
\begin{aligned}
& x \cdot \dot{V}^{\mathrm{ar}}(x \cdot l, l) \\
= & x \cdot \dot{\bar{V}}(x \cdot l, l)+(\partial \cdot \bar{V})(x \cdot l, l)+\eta(x \cdot l, l) \cdot[l \partial \cdot \bar{V}(x \cdot l, l)+x \cdot l \dot{\bar{V}}(x \cdot l, l)],
\end{aligned}
$$

and by identity (15) we obtain

$$
x \cdot A^{\operatorname{ar}}(x)=-\left.\frac{1}{2 \pi} \int \eta(s, l) \cdot[l \partial \cdot \bar{V}(s, l)+s \dot{\bar{V}}(s, l)]\right|_{s=x \cdot l} \mathrm{~d}^{2} l .
$$

The expression in square brackets in (53) is bounded by a constant, while $\eta(s, l)$ satisfies $(86)$, so by the use of estimate $(82)$ we find

$$
\begin{aligned}
\left|x \cdot A^{\operatorname{ar}}(x)\right| & \leq \text { const } \int \frac{\mathrm{d} \Omega(l)}{(1+|x \cdot l|)^{n}} \\
& \leq \frac{\mathrm{const}}{1+\left|x^{0}\right|+|\mathbf{x}|}\left\{\theta\left(-x^{2}\right)+\frac{\theta\left(x^{2}\right)}{\left(1+\left|x^{0}\right|-|\mathbf{x}|\right)^{n-1}}\right\} .
\end{aligned}
$$

In timelike directions the radial product $x \cdot A^{\text {ar }}(x)$ vanishes arbitrarily fast (depending on the choice of the function $\rho(x)$, see $(40)$ ). Moreover, let us introduce the scaling $\rho_{\delta}(a)=\delta^{-4} \rho(a / \delta)$, which for $\delta \searrow 0$ contracts the function to the central point, and denote by $A_{\delta}^{\text {ar }}$ the corresponding potential. Then $\eta_{\delta}(s, l)=\eta(s / \delta, l)$ and for $x \cdot A_{\delta}^{\mathrm{ar}}(x)$ the bound (54) holds with the replacement $x \rightarrow x / \delta$. Thus, outside $x=0$ this quantity vanishes with $\delta$ as

$$
\left|x \cdot A_{\delta}^{\mathrm{ar}}(x)\right| \leq \frac{\operatorname{const} \delta}{\delta+\left|x^{0}\right|+|\mathbf{x}|}\left\{\theta\left(-x^{2}\right)+\frac{\theta\left(x^{2}\right) \delta^{n-1}}{\left(\delta+\left|x^{0}\right|-|\mathbf{x}|\right)^{n-1}}\right\} .
$$

\subsection{Smearing with a Test Function}

Let $K^{b}(x)$ be a vector Schwartz test function (no conservation condition) and denote

$$
W(s, l)=\int_{M} K(x) \delta(s-x \cdot l) \mathrm{d} x
$$

which is a $C^{\infty}$-function, fast decaying in $s$, together with all its derivatives. Then

$$
A^{\mathrm{ar}}(K)=\int_{M} A^{\mathrm{ar}}(x) \cdot K(x) \mathrm{d} x=-\frac{1}{2 \pi} \int \dot{V}^{\mathrm{ar}}(s, l) \cdot W(s, l) \mathrm{d} s \mathrm{~d}^{2} l .
$$

Setting here (50) and integrating by parts (use (2) to transfer the derivative $\partial$ ), one finds

$$
\begin{aligned}
A^{\operatorname{ar}}(K) & =\frac{1}{2 \pi} \int \bar{V}(s, l) \cdot \dot{V}_{K}(s, l) \mathrm{d} s \mathrm{~d}^{2} l \\
& =-\frac{1}{2 \pi} \int \dot{V}(s, l) \cdot \bar{V}_{K}(s, l) \mathrm{d} s \mathrm{~d}^{2} l=\left\{\bar{V}_{K}, \bar{V}\right\}
\end{aligned}
$$

where

$$
\begin{aligned}
\dot{V}_{K}(s, l) & =\dot{W}(s, l)+\eta(s, l) l \cdot \dot{W}(s, l)+\zeta(s, l) \partial(l \cdot W(s, l)), \\
& =\dot{W}(s, l)+\partial_{s}[\eta(s, l) l \cdot W(s, l)]+\partial[\zeta(s, l) l \cdot W(s, l)], \\
\bar{V}_{K}(s, l) & =\frac{1}{2} \int_{\mathbb{R}} \operatorname{sgn}(s-\tau) \dot{V}_{K}(\tau, l) \mathrm{d} \tau .
\end{aligned}
$$


It follows that

$$
\begin{aligned}
l \cdot \bar{V}_{K}(s, l) & =0 \\
\Delta V_{K}(l) & =\partial \int_{\mathbb{R}} \zeta(s, l) l \cdot W(s, l) \mathrm{d} s .
\end{aligned}
$$

Orthogonality (58) is showed with the use of identity (85) as follows:

$$
\begin{aligned}
l \cdot \dot{V}_{K}(s, l) & =l \cdot \dot{W}(s, l)+(s \zeta(s, l)-1) l \cdot \dot{W}(s, l)+\zeta(s, l) l \cdot \partial(l \cdot W(s, l)) \\
& =\zeta(s, l)\left(s \partial_{s}+l \cdot \partial\right)(l \cdot W(s, l))=0,
\end{aligned}
$$

the last equality by the homogeneity of $l \cdot W(s, l)$. Equality (59) is immediate from the second form of $\dot{V}_{K}$ in (57).

We note that the function $\bar{V}_{K}(s, l)$ satisfies all demands (5) with $N=\infty$, (11) and (24) on a test function in the extended symplectic form (21), but in general - for non-conserved $K$-is outside the original test functions space.

\subsection{More General Smearing}

Now we want to extend the smearing $A^{\text {ar }}(K)$ to $C^{1}$-vector functions $K(x)$, with the spacetime asymptotic behavior characteristic for currents of scattered free particles or fields. We shall show that with appropriately specific conditions on $K$, the function $W(s, l)(55)$ is of class $C^{1}$ and satisfies the bounds

$$
|\dot{W}(s, l)| \leq \frac{\text { const }}{(1+|s|)^{1+\varepsilon}}, \quad|\partial[l \cdot W(s, l)]| \leq \frac{\text { const }}{(1+|s|)^{\varepsilon}} .
$$

Therefore, the integrals in (56), with the definitions (57), are absolutely convergent for this extension. Moreover, $\bar{V}_{K}(s, l)$ thus formed satisfies conditions (5) with $N=0,(11)$ and (24). In addition, let $g(x)$ be a Schwartz function, with $g(0)=1$ and denote $K_{\delta}(x)=g(\delta x) K(x)$. Then we shall find that irrespective of the shape of $g$ the following extension of the identity (56) holds true

$$
\begin{aligned}
A^{\operatorname{ar}}(K) \equiv \lim _{\delta \searrow 0} A^{\operatorname{ar}}\left(K_{\delta}\right) & =\frac{1}{2 \pi} \int \bar{V}(s, l) \cdot \dot{V}_{K}(s, l) \mathrm{d} s \mathrm{~d}^{2} l \\
& =-\frac{1}{2 \pi} \int \dot{V}(s, l) \cdot \bar{V}_{K}(s, l) \mathrm{d} s \mathrm{~d}^{2} l=\left\{\bar{V}_{K}, \bar{V}\right\} .
\end{aligned}
$$

We first note that once the existence of $W(s, l)$ with the properties $(60)$ is established, the existence of $\bar{V}_{K}(s, l)$ with the property (5) with $N=0$ and orthogonality (58) are obviously satisfied.

To specify the class for which the above results hold, it is convenient to characterize such class by splitting the function $K$ into three $C^{1}$-contributions: $K=K_{1}+K_{2}+K_{3}$, with the following properties:

$$
\begin{aligned}
& K_{1}(x)=x \kappa(x), \quad \text { supp } \kappa \subseteq\left\{x^{2} \geq 0,\left|x^{0}\right| \geq \frac{1}{2}\right\} \\
& \kappa(\lambda x)=\lambda^{-4} \kappa(x) \quad \text { for } \quad \lambda \geq 1,\left|x^{0}\right| \geq 1, \\
& |\kappa(x)| \leq \frac{\mathrm{const}}{(1+|x|)^{4}}, \quad|\partial \kappa(x)| \leq \frac{\mathrm{const}}{(1+|x|)^{5}}, \\
& \operatorname{supp} K_{2} \subseteq\left\{x^{2} \geq 0\right\}, \quad\left|K_{2}(x)\right| \leq \frac{\mathrm{const}}{(1+|x|)^{3}}, \quad \text { oscillatory part, }
\end{aligned}
$$




$$
\left|K_{3}(x)\right| \leq \frac{\text { const }}{(1+|x|)^{3+\varepsilon}}, \quad\left|\partial K_{3}(x)\right| \leq \frac{\text { const }}{(1+|x|)^{4+\varepsilon}} .
$$

Condition (62) characterizes the dominant asymptotic behavior of particles, and the non-oscillatory part of asymptotic behavior of fields. The oscillatory contribution in the case of fields is represented by $K_{2}$, bounded by (63), and we shall characterize it more precisely below. The rest is represented by $K_{3}$, conditions (64) characterizing the next to leading behavior inside the light cone, and decay of $K$ outside. ${ }^{9}$

We prove our claims separately for each contribution $K_{i}$, except for property (11), which will be discussed at the end.

We first note that all $W_{i}(s, l)$, defined by (55) with $K$ replaced by $K_{i}$, are absolutely convergent and bounded. Moreover, in case $i=3$ one has

$$
\left|W_{3}(s, l)\right|,\left|L_{a b} W_{3}(s, l)\right| \leq \frac{\text { const }}{(1+|s|)^{\varepsilon}}, \quad\left|\dot{W}_{3}(s, l)\right| \leq \frac{\text { const }}{(1+|s|)^{1+\varepsilon}}
$$

all these statements are proved as in Lemma 20 and Theorem 21 in ref. [15]. Therefore, bounds (60) are satisfied for $W_{3}$. Next, we observe that both

$$
A^{\mathrm{ar}}(x) \cdot K_{3}(x) \quad \text { and } A^{\mathrm{ar}}(x) \cdot K_{1}(x)=x \cdot A^{\mathrm{ar}}(x) \kappa(x)
$$

are absolutely integrable (see (52) and (54)), so the $\delta$-regularization in (61) is not needed for these contributions. Thus in case $i=3$ we obtain

$$
\begin{aligned}
A\left(K_{3}\right) & =-\frac{1}{2 \pi} \int_{M} \int \dot{V}^{\mathrm{ar}}(x \cdot l, l) \mathrm{d}^{2} l K_{3}(x) \mathrm{d} x \\
& =-\frac{1}{2 \pi} \int \dot{V}^{\mathrm{ar}}(s, l) \cdot W_{3}(s, l) \mathrm{d} s \mathrm{~d}^{2} l,
\end{aligned}
$$

so the thesis for this contribution follows.

In case $i=1$ we denote

$$
U(s, l)=\int_{M} \kappa(x) \delta(s-x \cdot l) \mathrm{d} x, \quad|U(s, l)| \leq \frac{\text { const }}{1+|s|}
$$

(the bound again by Lemma 20 in [15]), and observe that

$$
\partial U(s, l)=-\dot{W}_{1}(s, l), \quad s U(s, l)=l \cdot W_{1}(s, l) .
$$

Moreover, we note that the interior of the light cone (past and future) may be parametrized by $x=\lambda v, \lambda \in \mathbb{R}$ and $v$ on the future hyperboloid $v^{2}=1$,

\footnotetext{
${ }^{9}$ Suppose, for definiteness, that $K$ asymptotically behaves as the current of a free classical Dirac field. Then by standard stationary phase methods (e.g., [22]) the rest, beside the leading order in $K$, represented by $K_{3}$, is rather safely estimated by (64). The leading order in the asymptotic field, by this method, has the form of a homogeneous function of degree $-3 / 2$, with the support inside the light cone, multiplied by an oscillating term. In the hermitian form of the current, the oscillations cancel for terms with the same energy sign, and add up for terms with opposite energy sign. Proportionality of the homogeneous term to $x$ is related to the fact, that the current asymptotically is interpreted as particles escaping in timelike directions (see e.g., [11]). Matrix elements of the current will have similar structure in the quantum case.
} 
$v^{0} \geq 0$, and then $\mathrm{d} x=|\lambda|^{3} \mathrm{~d} \lambda \mathrm{d} \mu(v)$, where $\mathrm{d} \mu(v)=\mathrm{d}^{3} v / v^{0}$. It follows then from assumptions (62) that for $|s| \geq l^{0}$ one has

$$
\begin{aligned}
& W_{1}(s, l)=\int \operatorname{sgn}(\lambda) v \kappa(\operatorname{sgn}(\lambda) v) \delta(s-\lambda v \cdot l) \mathrm{d} \lambda \mathrm{d} \mu(v) \\
& =\operatorname{sgn}(s) \int \kappa(\operatorname{sgn}(s) v) \frac{v}{v \cdot l} \mathrm{~d} \mu(v) \\
& l \cdot W_{1}(s, l)=\operatorname{sgn}(s) \int \kappa(\operatorname{sgn}(s) v) \mathrm{d} \mu(v)
\end{aligned}
$$

Therefore,

$$
\dot{W}_{1}(s, l)=0, \quad \partial\left[l \cdot W_{1}(s, l)\right]=0 \quad \text { for } \quad|s| \geq l^{0} .
$$

We can now use (53) to obtain (we omit the arguments $(s, l)$ for the sake of clarity)

$$
\begin{aligned}
A\left(K_{1}\right) & =-\frac{1}{2 \pi} \int \eta \cdot[l \partial \cdot \bar{V}+s \dot{\bar{V}}] U \mathrm{~d} s \mathrm{~d}^{2} l \\
& =-\frac{1}{2 \pi} \int\left[s U \partial_{s}(\bar{V} \cdot \eta)+(1-s \zeta) \bar{V} \cdot \partial U\right] \mathrm{d} s \mathrm{~d}^{2} l
\end{aligned}
$$

where for the second equality we integrated $\partial$ by parts and used the equality $\partial(l \cdot \eta)=-s \dot{\zeta}$, which follows from identities (84) and (85). Performing now the substitutions (65) (for $s U$ using the second of these relations in both places where it appears) and integrating $\partial_{s}$ by parts, we obtain

$$
A\left(K_{1}\right)=\frac{1}{2 \pi} \int \bar{V} \cdot\left[\dot{W}_{1}+\eta l \cdot \dot{W}_{1}+\zeta \partial\left(l \cdot W_{1}\right)\right] \mathrm{d} s \mathrm{~d}^{2} l,
$$

which is the required result.

We turn to the case $i=2$. The decay of $K_{2}$ is not sufficient to apply the proof presented in case $i=3$. However, the oscillatory behavior of $K_{2}$ damps the integrals, and we now add further assumption that $W_{2}$ satisfies bounds as in case $W_{3}$ :

$$
\left|W_{2}(s, l)\right|,\left|L_{a b} W_{2}(s, l)\right| \leq \frac{\text { const }}{(1+|s|)^{\varepsilon}}, \quad\left|\dot{W}_{2}(s, l)\right| \leq \frac{\text { const }}{(1+|s|)^{1+\varepsilon}} .
$$

We denote

$$
W_{2 \delta}(s, l)=\int_{M} g(\delta x) K_{2}(x) \delta(s-x \cdot l) \mathrm{d} x,
$$

and assume further that

$$
\left|W_{2 \delta}(s, l)\right| \leq \frac{\text { const }}{(1+|s|)^{\varepsilon}}
$$

With these assumptions, we have

$$
\begin{aligned}
A\left(K_{2 \delta}\right) & =\int \dot{V}^{\mathrm{ar}}(s, l) \cdot W_{2 \delta}(s, l) \mathrm{d} s \mathrm{~d}^{2} l \\
& \rightarrow \int \dot{V}^{\mathrm{ar}}(s, l) \cdot W_{2}(s, l) \mathrm{d} s \mathrm{~d}^{2} l \quad \text { for } \quad \delta \searrow 0
\end{aligned}
$$


the limit by the dominated convergence theorem. This, together with the estimates (67), leads to the thesis. We show in Appendix E that our assumptions on $W_{2}$ are satisfied for a term of the type characteristic for the Dirac field.

Finally, we shall close the proof of our claims by showing (11), namely

$$
\Delta V_{K}(l)=-\partial \Phi_{K}(l),
$$

with

$$
\begin{aligned}
\Phi_{K}(l) & =\int[\kappa(v)+\kappa(-v)] \rho(a) \log \frac{|a \cdot l|}{v \cdot l} \mathrm{~d} \mu(v) \mathrm{d} a \\
& -\int \zeta(s, l) l \cdot[W(s, l)-W(\operatorname{sgn}(s) \infty, l)] \mathrm{d} s .
\end{aligned}
$$

First, we observe that it is only $W_{1}$ that contributes to $W( \pm \infty, l)$, so by $(66)$ we have

$$
W( \pm \infty, l)= \pm \int \kappa( \pm v) \frac{v}{v \cdot l} \mathrm{~d} \mu(v), \quad l \cdot W( \pm \infty, l)= \pm \int \kappa( \pm v) \mathrm{d} \mu(v) \equiv Q_{ \pm} .
$$

Differentiating the first line in (71) we obtain

$$
\begin{aligned}
\int[\kappa(v)+\kappa(-v)] \rho(a)\left[\frac{a}{a \cdot l}\right. & \left.-\frac{v}{v \cdot l}\right] \mathrm{d} \mu(v) \mathrm{d} a \\
& =\eta(0, l)\left(Q_{-}-Q_{+}\right)-W(+\infty, l)+W(-\infty, l) .
\end{aligned}
$$

Differentiation of the second line in $(71)$ gives

$$
-\int \partial[\zeta(s, l) l \cdot W(s, l)] \mathrm{d} s+\int \partial \zeta(s, l) Q_{\operatorname{sgn}(s)} \mathrm{d} s .
$$

Using (84) in the second integral and summing the contributions, we obtain

$$
\partial \Phi_{K}(l)=-W(+\infty, l)+W(-\infty, l)-\int \partial[\zeta(s, l) l \cdot W(s, l)] \mathrm{d} s,
$$

which substituted into (70) gives the correct value. Note that the differential $\partial$ cannot be extracted outside the integral in the last formula, as without it the integrand is not integrable.

\subsection{Quantum Field}

We have shown in the classical case that $A^{\text {ar }}(K)=\left\{\bar{V}_{K}, \bar{V}\right\}$. However, recall the identity (31), which holds in each of the cases (26), that is both for 'in' and for 'out' algebra. Thus, on the algebraic level, in each of these two cases we propose to define the quantum almost radial gauge of the potential by ${ }^{10}$

$$
A^{\mathrm{ar}}(K)=\left\{V_{K}, V^{\mathrm{q}}\right\},
$$

whenever $V_{K}(s, l)$ defined by (57) and (26) falls in our extended symplectic space defined after (27). This condition demands stronger regularity properties of $K$ than those assumed in Section 5.4, leading to classical version of (72). One can show that a class of such functions may be obtained by a modification of the currents $J$ considered in [13], where the modification consists in demanding

\footnotetext{
${ }^{10}$ Our extended field algebra of Section 3 is defined directly in terms of test functions $V(s, l)$, and in that sense the definition (72) is fully compatible with its classical prototype. We do not consider here the question of convergence of the quantum version of the limit (61).
} 
the continuity equation to be satisfied only asymptotically in time (in the form of an appropriately regular analogue of (62)). On the other hand, in concrete representations the scope of admitted test functions is wider, and the definition of the almost radial gauge may be also appropriately extended at the level of representation. We do not go into details here and leave the question to be decided in applications.

The almost radial gauge is a Lorenz gauge. For $K(x)=\partial F(x)$ one finds that $V_{K}(s, l)=W(s, l) \propto l$, which is in the zero equivalence class. Thus

$$
A^{\operatorname{ar}}(\partial F)=0 .
$$

Moreover, for $K(x)=\square G(x)$ we have $W(s, l)=0$, so

$$
A^{\mathrm{ar}}(\square G)=0 \text {. }
$$

For test functions $K(x)$ in the class of conserved currents admitted in $A(K)$ in one of the cases 'in' or 'out,' one has $l \cdot W(s, l)=0$, so that $\dot{V}_{K}(s, l)=$ $\dot{W}(s, l)$ and $V_{K}(s, l)=W(s, l)$. Therefore, in this case

$$
A^{\operatorname{ar}}(K)=A(K),
$$

which reproduces the extended free algebra.

Finally, let us note the following important corollary. If $K$ is a conserved, non-radiating current, such as the current of a free, charged classical particle, or of the free (classical or quantum) Dirac field, then $W(s, l)=W(l)$ is constant in $s$ and $l \cdot W(l)=Q$, the charge of the current (see ref. [11], discussion starting with Eq. (2.70)). Therefore, for such currents $V_{K}=0$ and

$$
A^{\operatorname{ar}}(K)=0 \text {. }
$$

\subsection{Transformation and Commutation Properties}

We recall that almost radial gauge is a functional of the function $\rho$ serving to smear the point $a$ from which the radial line to $x$ is drawn. Let us make this dependence explicit by writing $A^{\text {ar }}(K ; \rho)$. With Poincaré transformations of the extended algebra defined in (28), these elements have the covariance property given by

$$
\alpha_{z, \Lambda}\left[A^{\operatorname{ar}}(K ; \rho)\right]=A^{\operatorname{ar}}\left(T_{z, \Lambda} K ; T_{z, \Lambda} \rho\right) .
$$

In this sense $A(K ; \rho)$ may be regarded algebraically covariant, with unitarily implementable translations. For the proof of the covariance relation one notes that

$$
\begin{aligned}
W\left[T_{z, \Lambda} K\right](s, l) & =\Lambda W[K]\left(s-z \cdot l, \Lambda^{-1} l\right), \\
\zeta\left[T_{z, \Lambda} \rho\right](s, l) & =\zeta[\rho]\left(s-z \cdot l, \Lambda^{-1} l\right), \\
\eta\left[T_{z, \Lambda} \rho\right](s, l) & =\Lambda \eta[\rho]\left(s-z \cdot l, \Lambda^{-1} l\right)+z \zeta[\rho]\left(s-z \cdot l, \Lambda^{-1} l\right) .
\end{aligned}
$$

Setting these relations into Formula (57) for $V_{K}$, and then using the result in (29), one arrives at the thesis.

Elements $A^{\text {ar }}(K ; \rho)$ do not have compact localization even if the support of $K$ is compact. However, the following remnant timelike locality with respect to $A$ holds. Let the support of an admissible test vector function $K$ and the 
support of a conserved test current $J$ be timelike separated, and let $V(s, l)$ for current $J$ (16) have $\Delta V(l)=0$ (in particular, this is always true for compactly supported $J)$. Then

$$
\left[A^{\operatorname{ar}}(K ; \rho), A(J)\right]=0,
$$

irrespective of the choice of $\rho$. To show this, we first note that it is sufficient to show this when the two supports are placed inside the future and the past parts of the same light cone, with any vertex point $b$. Next, we apply to the commutator translation automorphism $\alpha_{-b, \mathbb{1}}$. The commutator, without changing its value (being proportional to identity), takes now the form $\left[A^{\operatorname{ar}}\left(K^{\prime} ; \rho^{\prime}\right), A\left(J^{\prime}\right)\right]$, where $K^{\prime}$ and $J^{\prime}$ are similarly separated as $K$ and $J$, but with the vertex of the light cone in the origin. In consequence, the functions $W^{\prime}(s, l)$ for $K^{\prime}(55)$, and $V^{\prime}(s, l)$ for $J^{\prime}(16)$, are supported in $s \in(0,+\infty)$ or $s \in(-\infty, 0)$, each in a different of the two sets. Now, it follows from (57) that the support of $\dot{V}_{K^{\prime}}(s, l)$ is not larger than the support of $W^{\prime}(s, l)$, so the same conclusion is valid for the pair $\dot{V}_{K^{\prime}}(s, l)$ and $V^{\prime}(s, l)$. But from $\Delta V^{\prime}(l)=0$, together with (26), it follows that

$$
\left\{V_{K^{\prime}}, V^{\prime}\right\}=\frac{1}{2 \pi} \int_{\mathbb{R}} \dot{V}_{K^{\prime}}(s, l) \cdot V^{\prime}(s, l) \mathrm{d} s \mathrm{~d}^{2} l,
$$

so the thesis follows.

\section{Almost Radial Gauge and String-Localized Fields: Comparative Remarks}

Definition of the almost radial gauge was motivated by integration, on the classical level, of the electromagnetic potential along straight axes (43). Another construction based on integration along infinitely extended straight lines is the formalism of string-localized fields (potentials) developed by Mund, Schroer and Yngvason [21] (based on earlier works, as explained in this reference) and worked out later in many articles. ${ }^{11}$ The two constructions result from quite different motivations and aims; nevertheless, it seems interesting to make some comparison. For that purpose, we briefly recall and formulate in our language the latter in the case of electrodynamics. ${ }^{12}$ All electromagnetic fields are assumed to satisfy the homogeneous Maxwell equations.

The string-localized potentials in electrodynamics are distributions in position vectors $x$ and spacelike unit vectors $e$ defined by ${ }^{13}$

$$
A_{b}^{\mathrm{sl}}(x, e)=\int_{\mathbb{R}_{+}} F_{b a}(x+\lambda e) e^{a} \mathrm{~d} \lambda .
$$

\footnotetext{
${ }^{11}$ See e.g., a recent discussion of an application to QED problems [20].

${ }^{12}$ Our perspective is different from the discussion of string-localized fields in [21], which starts from the unitary representations of the Poincaré group and engages the notion of modular localization [2]. Here we want to avoid the a priori assumption on the action of a representation of the Lorentz group.

${ }^{13}$ There exists a version of this construction with lightlike $e$, see [10].
} 
To obtain a vector potential as an $x$-distribution, one needs a scalar test function $h(e)$ on the hyperboloid $e^{2}=-1$, such that

$$
\int h(e) \mathrm{d} \nu(e)=1
$$

where the integration is over the hyperboloid with the Lorentz invariant measure $\mathrm{d} \nu(e)$. Then,

$$
A_{b}^{\mathrm{sl}}(x ; h)=\int A_{b}^{\mathrm{sl}}(x, e) h(e) \mathrm{d} \nu(e)
$$

is an $h$-dependent potential, commuting with the field $F_{a b}(y)$ at points $y$ in spacelike position to the set of points $x+\lambda e, \lambda \in \mathbb{R}_{+}, e \in \operatorname{supp} h$. In particular, if $\operatorname{supp} h$ is contained in some double-cone spacelike separated from 0 , then $A^{\mathrm{sl}}(x ; h)$ is localized in a spacelike cone.

This potential is in the Lorenz class, and using the representation (12) we show in Appendix $\mathrm{F}$ that on the classical level

$$
A_{b}^{\mathrm{sl}}(x ; h)=-\frac{1}{2 \pi} \int \dot{V}_{b}^{\mathrm{sl}}(x \cdot l, l) \mathrm{d}^{2} l,
$$

where

$$
\begin{aligned}
\dot{V}^{\mathrm{sl}}(s, l) & =\dot{V}(s, l)-l n(l) \cdot \dot{V}(s, l)-l z(l) \cdot \int \frac{\dot{V}(\tau, l)}{s-\tau} \mathrm{d} \tau, \\
n(l) & =\int \frac{e h(e)}{e \cdot l} \mathrm{~d} \nu(e), \quad z(l)=\int e h(e) \delta(e \cdot l) \mathrm{d} \nu(e),
\end{aligned}
$$

where the integrals in (78) and in $n(l)$ are in the principal value sense, and not to burden notation we do not indicate the dependence on $h$ explicitly. For a vector Schwartz test function $K(x)$ we then find in analogy to (56)

$$
A^{\mathrm{sl}}(K ; h)=\left\{V_{K}^{\mathrm{sl}}, V\right\}
$$

where in place of (57), and keeping notation (55), we have

$$
V_{K}^{\mathrm{sl}}(s, l)=W(s, l)-n(l) l \cdot W(s, l)+z(l) \int \frac{l \cdot W(\tau, l)}{s-\tau} \mathrm{d} \tau .
$$

In contrast to $W(s, l)$, which is of fast decrease, $V_{K}^{\mathrm{sl}}(s, l)$ vanishes in general only as $s^{-1}$, see the last term in (80). Therefore, the (exponentiated) formula (79) cannot give, upon quantization, an element in the local algebra, even if $K$ is local. However, we have

$$
\widetilde{V}_{K}^{\mathrm{sl}}(\omega, l)=\widetilde{W}(\omega, l)-n(l) l \cdot \widetilde{W}(\omega, l)+i \pi z(l) \operatorname{sgn}(\omega) l \cdot \widetilde{W}(\omega, l),
$$

which is sufficient for elements (79) to be represented in the standard vacuum representation of the field $F$, in contrast to the almost radial gauge, which needs the extended algebra including infrared singular fields.

The formulation of a gauge theory in terms of string-localized potentials puts stress on their Poincaré covariance. In vacuum representation the action of the Poincaré automorphisms $\alpha_{z, \Lambda}$ of the observable algebra given by (28) is 
implemented by a unitary representation $U(z, \Lambda)$. Then, in this representation, one also has

$$
\alpha_{z, \Lambda}\left[A^{\mathrm{sl}}(K ; h)\right]=A^{\mathrm{sl}}\left(T_{z, \Lambda} K ; T_{0, \Lambda} h\right)=U(z, \Lambda) A^{\mathrm{sl}}(K ; h) U(z, \Lambda)^{*} .
$$

At the algebraic level our transformation law (73) is analogous to (81). However, as mentioned before, in our case not all Lorentz transformations are implemented.

Let us now return again to the question of localization and commutation. String-localized fields located on spacelike separated strings commute (which remains true for slightly smeared strings); this is an important property in the formalism of these fields. Whether almost radial gauge may be localized in spacelike extended regions in some special cases and special points $x$ is a subtle question, which we do not want to consider here; anyway, this cannot be a generic property of this gauge. On the other hand, it has a timelike commutation property (74). We have seen that this property depended on the fact that the support of $\dot{V}_{K}(s, l)$ was not greater than that of $W(s, l)$. The latter property does not hold for $V_{K}^{\text {sl }}(s, l)$ because of the last term in $(80)$. Therefore, for string-localized fields timelike commutativity does not hold, in general.

It is interesting to note that the function $z(l)$ vanishes for even functions $h, h(-e)=h(e)$, which are admitted by the condition (75). For such functions the last term in $(80)$ is absent and the elements $A^{\text {sl }}(K ; h)$ acquire the timelike locality as formulated above for $A^{\text {ar }}(K ; \rho)$. As here the algebra is the standard local electromagnetic algebra of the free field, this leads in fact to the following implication in the vacuum representation. If the support of the smearing function $K$ is contained in an open double-cone with vertices $x_{1}$ and $x_{2}, x_{2}^{0}>x_{1}^{0}$, then $A^{\mathrm{sl}}(K)$ belongs to the intersection of the von Neumann algebras connected with the following two regions: the interior of the future light cone with the vertex in $x_{1}$, and the interior of the past light cone with the vertex in $x_{2}$. This follows from the timelike duality for the vacuum representation of massless fields, see [5]. Condition of evenness of $h$ may be equivalently formulated as string integration along whole real axes, instead of semi-axes; the spacelike localization and commutation properties are then retained (with obvious modifications). ${ }^{14}$

One could also observe that the last term in (80) seems to worsen the infrared/adiabatic limit behavior: for $K$ becoming infinitely extended so that $W(s, l)$ does not vanish in infinity, this term becomes meaningless. On the other hand, if the term is absent, then the algebra of elements (79) may be also built in the extended algebra of electromagnetic field described earlier.

\footnotetext{
${ }^{14}$ The relation with the notion of modular localization, as used in the string-localized formalism of [21], is then lost, or at least becomes indirect.
} 


\section{Outlook}

The results of the analysis in $[11,15]$ suggest that, for the classical MaxwellDirac system in an appropriate radial-like gauge, the asymptotic Dirac and radiation fields may be largely separated, with the Dirac field carrying its own Coulomb field. Motivated by this, we proposed in $[12,13]$ an asymptotic algebra incorporating these ideas on the quantum level. The free electromagnetic part of this algebra is the extended algebra described in Section 3. In order to turn on full interaction in this setting, one needs an appropriate potential. The present construction is a further step in our program.

One should stress that our almost radial gauge could not be constructed within the limits of the standard local theory: see remarks at the end of Section 5.3 and the definition of the quantum almost radial gauge in Section 5.5. Our construction engages nonlocal quantum variables of the extended algebra, which do not appear in the local theory (either in its $C^{*}$-structure, or in its irreducible representations). Therefore, these nonlocal aspects are not a consequence, but a prerequisite of the present construction. The long-range structure of the representations of our asymptotic algebra, quite different from the structure in local quantum electrodynamics, was partly considered in $[13,14]$. Further investigation of these questions, not being directly related to the construction of the almost radial gauge, is left for a discussion elsewhere.

\section{Acknowledgements}

I am grateful to Paweł Duch, Wojciech Dybalski and José M. Gracia-Bondía for reading the manuscript and for interesting correspondence. Also, some editorial remarks from the Referees are gratefully acknowledged.

Open Access. This article is licensed under a Creative Commons Attribution 4.0 International License, which permits use, sharing, adaptation, distribution and reproduction in any medium or format, as long as you give appropriate credit to the original author(s) and the source, provide a link to the Creative Commons licence, and indicate if changes were made. The images or other third party material in this article are included in the article's Creative Commons licence, unless indicated otherwise in a credit line to the material. If material is not included in the article's Creative Commons licence and your intended use is not permitted by statutory regulation or exceeds the permitted use, you will need to obtain permission directly from the copyright holder. To view a copy of this licence, visit http://creativecommons. org/licenses/by/4.0/.

Publisher's Note Springer Nature remains neutral with regard to jurisdictional claims in published maps and institutional affiliations. 


\section{Appendix}

\section{A. Estimates}

Here we note the following estimates. For $a, b, c, \alpha$, all $>0$, one has

$$
\int_{0}^{c} \frac{\mathrm{d} u}{(a+b u)^{\alpha}} \leq \begin{cases}\frac{\alpha}{\alpha-1} \frac{c}{a^{\alpha-1}(a+b c)}, & \alpha>1, \\ \frac{1}{1-\alpha} \frac{c}{(a+b c)^{\alpha}}, & \alpha<1, \\ \frac{2 c}{a+b c} \log \left(e+\frac{b c}{a}\right), & \alpha=1,\end{cases}
$$

with Euler's number $e$. Cases $\alpha \lessgtr 1$ may be found in [11], Appendix B, and are reproduced here for convenience of the reader. In case $\alpha=1$ the result of integration is $\frac{1}{b} \log \left(1+\frac{b c}{a}\right)$. For $b c \leq a$ this is bounded by $\frac{c}{a} \leq \frac{2 c}{a+b c}$, while for $b c \geq a$ by $\frac{2 c}{a+b c} \log \left(1+\frac{b c}{a}\right)$.

\section{B. Function $r^{a}(x, z)$}

Here we discuss properties of function $r^{a}(x, z)$ defined in (41), and we start with its estimate. We denote by $x_{z}$ and $x_{\perp}$ the parallel and the perpendicular (in Euclidean metric) to $z$ components of $x$, respectively. Then for each $k \in \mathbb{N}$ we have

$$
\left|r^{a}(x, z)\right| \leq \operatorname{const}(k) \frac{\left(1+\left|x_{z}\right|\right)^{3}}{\left(1+\left|x_{\perp}\right|\right)^{k}} \frac{1}{|z|^{3}} .
$$

To show this, we use $|\rho(x)| \leq \operatorname{const}(1+|x|)^{-k-4}$ to estimate the rhs of (41) by

$$
\text { const } \int_{\mathbb{R}} \frac{|z||u|^{3} \mathrm{~d} u}{\left(1+\left|x_{\perp}\right|+|u| z\left|+x_{z}\right|\right)^{k+4}} \leq \frac{\text { const }}{|z|^{3}} \int_{0}^{\infty} \frac{\left(r+\left|x_{z}\right|\right)^{3} \mathrm{~d} r}{\left(1+\left|x_{\perp}\right|+r\right)^{k+4}},
$$

which leads easily to (83).

Next, we prove identity (42). We observe that outside $z=0$ the function $\int \rho(x+u z)|u|^{3} \mathrm{~d} u$ is smooth and homogeneous in $z$ of degree -4 , so the distribution $\partial^{z} \cdot r(x, z)$ is concentrated in $z=0$. We choose a $C^{1}$-function $f(z)$ which together with its derivative decays at least as $|z|^{-2}$, denote $\hat{z}=z /|z|$ and write $\mathrm{d} S(\hat{z})$ for the integration element on the (Euclidean) unit sphere. Then

$$
\begin{aligned}
& -\int_{M} r(x, z) \cdot \partial f(z) \mathrm{d} z=-\lim _{\epsilon \backslash 0} \int_{M} r(x, z) \cdot \partial f(z) \theta(|z|-\epsilon) \mathrm{d} z \\
& =\frac{1}{2} \lim _{\epsilon \searrow 0} \int_{M} \int_{\mathbb{R}} \rho(x+u z)|u|^{3} \mathrm{~d} u f(z) \delta(|z|-\epsilon)|z| \mathrm{d} z \\
& =\frac{1}{2} \lim _{\epsilon \searrow 0} \int_{S^{3}} \int_{\mathbb{R}} \rho(x+r \hat{z})|r|^{3} \mathrm{~d} r f(\epsilon \hat{z}) \mathrm{d} S(\hat{z})=f(0) \int_{M} \rho(x+z) \mathrm{d} z=f(0),
\end{aligned}
$$

where the third equality is obtained by the change of variables $r=\epsilon u$. This ends the proof. 


\section{Functions $\zeta$ and $\eta$}

The functions $\zeta$ and $\eta$ defined by (46) are smooth outside $l=0$ and homogeneous of degree -1 with respect to their arguments, and satisfy the following identities:

$$
\begin{aligned}
& \partial \zeta(s, l)+\dot{\eta}(s, l)=0, \\
& s \zeta(s, l)=1+l \cdot \eta(s, l) .
\end{aligned}
$$

The Fourier transforms are

$$
\widetilde{\zeta}(\omega, l)=i \pi \operatorname{sgn}(\omega) \hat{\rho}(\omega l), \quad \widetilde{\eta}(\omega, l)=\pi \operatorname{sgn}(\omega)(\partial \hat{\rho})(\omega l) .
$$

Conditions (40) are equivalent to

$$
\hat{\rho}(0)=\frac{1}{2 \pi}, \quad \partial^{\alpha} \hat{\rho}(0)=0 \quad \text { for } \quad 1 \leq|\alpha| \leq n,
$$

which implies that $\widetilde{\eta}(\omega, l)$ is $n-1$ times continuously differentiable and

$$
\partial_{\omega}^{k} \widetilde{\eta}(\omega, l)_{\mid \omega=0}=0, \quad k=0, \ldots, n-1,
$$

while $\partial_{\omega}^{n} \widetilde{\eta}(\omega, l)$ is bounded and integrable. It follows that

$$
|\eta(s, l)| \leq \frac{\text { const }}{(1+|s|)^{n}} .
$$

\section{Derivation of $S^{\text {ar }}(x)$ for Free Field}

Here we prove Formula (48). Formula (47) for $S_{\delta}^{\text {ar }}(x)$ may be interpreted as the definition (45) with $\chi(\delta z) A(x-z)$ substituted for $A(x-z)$. Equivalently, this is expression (39) with $\chi(-\delta \xi \overrightarrow{a x}) A(x+\xi \overrightarrow{a x})$ substituted for $A(x+\xi \overrightarrow{a x})$, and smeared with $\rho(a)$. We change integration variables from $a$ to $z=a-x$, multiply the expression for convenience by $2 \pi$, and obtain

$$
2 \pi S_{\delta}^{\mathrm{ar}}(x)=-\frac{1}{2} \int_{M} \int_{\mathbb{R}} \chi(\delta \xi z) \rho(x+z) \int z \cdot \dot{V}(x \cdot l-\xi z \cdot l, l) \mathrm{d}^{2} l \operatorname{sgn}(\xi) \mathrm{d} \xi \mathrm{d} z .
$$

We note that the integrand is absolutely integrable so we can freely change the order of integration and leave the $\mathrm{d}^{2} l$ integral to be performed at the last stage. In the remaining integrals we change $\xi$ to $\lambda=\delta \xi$ and reduce integration with respect to $\lambda$ to the positive semi-axis by reflecting $\lambda \rightarrow-\lambda$ from the negative semi-axis. The result may be written as

$$
-\int_{M} \int_{\mathbb{R}_{+}} \chi(\lambda z) \rho(x+z) \frac{\partial}{\partial \lambda} \frac{z \cdot Z\left(x \cdot l, \delta^{-1} \lambda z \cdot l, l\right)}{z \cdot l} \mathrm{~d} \lambda \mathrm{d} z,
$$

where

$$
Z(\tau, u, l)=\bar{V}(\tau, l)-\frac{1}{2}[\bar{V}(\tau+u, l)+\bar{V}(\tau-u, l)] .
$$

We note that $Z(\tau, u, l)$ is bounded, $Z(\tau, 0, l)=0$, and

$$
\lim _{\delta \rightarrow 0} Z\left(x \cdot l, \delta^{-1} \lambda z \cdot l, l\right)=\bar{V}(x \cdot l, l) \quad \text { almost everywhere, }
$$

with the choice of $\bar{V}(s, l)$ according to Formula (7).

We now submit (87) to the following transformations:

- integration with respect to $\lambda$ by parts; 
- replacement of $\rho(x+z)$ by $\nu(x, z)=\frac{1}{2}[\rho(x+z)+\rho(x-z)]$, possible due to the evenness in $z$ of the combined remaining factors;

- choice of variables $w^{a}$ for vector $z$, such that $w^{0}=z \cdot l, \mathrm{~d} z=\mathrm{d} w^{0} \mathrm{~d}^{3} w_{\perp}$, with $w_{\perp}=\left(w^{1}, w^{2}, w^{3}\right)$.

In this way (87) takes the form

$$
\int_{\mathbb{R}} \int_{\mathbb{R}_{+}}\left(w^{0}\right)^{-1} f\left(x, \lambda, w^{0}\right) \cdot Z\left(x \cdot l, \delta^{-1} \lambda w^{0}, l\right) \mathrm{d} \lambda \mathrm{d} w^{0},
$$

where

$$
f\left(x, \lambda, w^{0}\right)=\int_{\mathbb{R}^{3}} \frac{\partial}{\partial \lambda} \chi(\lambda w) \nu(x, w) w \mathrm{~d} w_{\perp} .
$$

It is easy to see that $f\left(x, \lambda, w^{0}\right)$ is a $C^{\infty}$-function, and $f(x, \lambda, 0)=0$ due to the oddness of the integrand in $w$, so $\left(w^{0}\right)^{-1} f\left(x, \lambda, w^{0}\right)$ is also $C^{\infty}$. An easy calculation also shows that the latter function is absolutely integrable with respect to $\mathrm{d} \lambda \mathrm{d} w^{0}$. Therefore, we can take the $\delta \rightarrow 0$ limit under all integrations and the limit of (88) becomes

$$
\bar{V}(x \cdot l, l) \cdot \int_{\mathbb{R}} \int_{\mathbb{R}_{+}} f\left(x, \lambda, w^{0}\right)\left(w^{0}\right)^{-1} \mathrm{~d} \lambda \mathrm{d} w^{0}=-\bar{V}(x \cdot l, l) \cdot \int_{\mathbb{R}^{4}} \frac{w \nu(x, w)}{w^{0}} \mathrm{~d} w,
$$

in the latter form integral in the principal value sense. We can now restore back the abstract notation of vector $z$, and the function $\rho(x+z)$ in place of $\nu(x, z)$ (due to the evenness of $\left(w^{0}\right)^{-1} w$ ). We finally restore the integration variable $a=x+z$ and then

$$
-\int_{\mathbb{R}^{4}} \frac{\nu(x, w) w}{w^{0}} \mathrm{~d} w=\int_{M} \frac{\rho(a)(a-x)}{x \cdot l-a \cdot l} \mathrm{~d} a=\eta(x \cdot l, l)-x \zeta(x \cdot l, l) .
$$

\section{E. Oscillatory Asymptotic Term}

Here we make more specific assumptions on oscillating smearing function $K_{2}$ introduced in Section 5.4. Function $K_{2}$ specified below obviously satisfies (63), and we shall show that assumptions (67) and (69) are satisfied with $\varepsilon=1$.

Let $K_{2}(x)$ be of the form (cf. [11])

$$
K_{2}(x)=e^{i \mu \sqrt{x^{2}}} k(x), \quad \mu>0,
$$

where $k(x)$ is a $C^{2}$-vector function, supported inside the light cone and vanishing for $\left|x^{0}\right| \leq 1$. Moreover, we assume that in the parametrization $x=\lambda v$ introduced after (65) the following bounds are satisfied:

$$
\left|\left(\partial^{\alpha} k\right)(\lambda v)\right| \leq \frac{\text { const }}{\lambda^{3+|\alpha|}\left(v^{0}\right)^{n}}, \quad|\alpha| \leq 2 .
$$

where for simplicity we assume that $n$ may be arbitrarily large; by a rather tedious control of the decay in $v$, this could be substantially weakened, but we do not need this for our illustrative purposes.

For $W_{2 \delta}$ formed as in (68) we have

$$
W_{2 \delta}(s, l)=\int e^{i \mu \lambda} k(\lambda v) g(\delta \lambda v) \delta(s-\lambda v \cdot l)|\lambda|^{3} \mathrm{~d} \lambda \mathrm{d} \mu(v) .
$$


We integrate with respect to $\lambda$, which gives

$$
W_{2 \delta}(s, l)=\int e^{i \mu \lambda} k(\lambda v) g(\delta \lambda v)|\lambda|^{3} \frac{\mathrm{d} \mu(v)}{v \cdot l},
$$

where from now on we put $\lambda=\lambda(s, v, l)=s / v \cdot l$. It is clear that $\left|W_{2 \delta}(s, l)\right|$ is bounded by a constant independent of $\delta$, and

$$
\lim _{\delta \rightarrow 0} W_{2 \delta}(s, l)=W_{2}(s, l)=\int e^{i \mu \lambda} k(\lambda v)|\lambda|^{3} \frac{\mathrm{d} \mu(v)}{v \cdot l} .
$$

To proceed, we need an integral identity on the hyperboloid $v^{2}=1$, $v^{0}>0$. Consider operators

$$
M_{a b}=v_{a} \frac{\partial}{\partial v^{b}}-v_{b} \frac{\partial}{\partial v^{a}}, \quad \delta_{b}=v^{a} M_{a b}=\frac{\partial}{\partial v^{b}}-v_{b} v \cdot \frac{\partial}{\partial v},
$$

intrinsic differentiation operators in the hyperboloid. The measure $\mathrm{d} \mu(v)$ is Lorentz invariant, and operators $M_{a b}$ generate Lorentz transformations in the hyperboloid, so if $\chi(v)$ and $M_{a b} \chi(v)$ are absolutely integrable, then

$$
\int M_{a b} \chi(v) \mathrm{d} \mu(v)=0 .
$$

Moreover, we have $M_{a b} v^{a} \chi(v)=\left(\delta_{b}-3 v_{b}\right) \chi(v)$. Therefore, if

$$
|\chi(v)| \leq \frac{\text { const }}{\left(v^{0}\right)^{3+\varepsilon}}, \quad|\delta \chi(v)| \leq \frac{\text { const }}{\left(v^{0}\right)^{2+\varepsilon}},
$$

then

$$
\int(\delta-3 v) \chi(v) \mathrm{d} \mu(v)=0
$$

We note the identities and a bound

$$
l \cdot \delta \frac{1}{v \cdot l}=1, \quad e^{i \mu \lambda}=-\frac{i}{s \mu} l \cdot \delta e^{i \mu \lambda}, \quad l \cdot \delta \frac{v}{v \cdot l}=\frac{l}{v \cdot l}, \quad \frac{1}{v \cdot l} \leq \frac{2 v^{0}}{l^{0}} .
$$

Substituting the second of these relations in (90) and integrating by parts by (92) (our assumptions on decay of $k$ and $\partial k$ make it possible), we find

$$
\begin{aligned}
W_{2 \delta}(s, l) & =\frac{i}{\mu s} \int e^{i \mu \lambda} g(\delta \lambda v)|\lambda|^{3}\left(k(\lambda v)+\frac{\lambda}{v \cdot l} l \cdot \partial k(\lambda v)\right) \mathrm{d} \mu(v), \\
& +\frac{i \delta}{\mu s} \int e^{i \mu \lambda}|\lambda|^{3} k(\lambda v) \frac{\lambda}{v \cdot l} l \cdot \partial g(\delta \lambda v) \mathrm{d} \mu(v) .
\end{aligned}
$$

We note that $|g(\delta \lambda v)|$ is bounded by a constant and tends to 1 for $\delta \rightarrow 0$, while $\delta|\lambda|\left|v^{0}\right||\partial g(\delta \lambda v)|$ is also bounded by a constant and tends to zero in that limit. Thus, using (89) we find that $\left|W_{2 \delta}(s, l)\right| \leq$ const $|s|^{-1}$, so the bound (69) and the first of the bounds (67) are satisfied with $\varepsilon=1$. Moreover, taking the limit we find

$$
W_{2}(s, l)=\frac{i}{\mu s} \int e^{i \mu \lambda}|\lambda|^{3}\left(k(\lambda v)+\frac{|\lambda|}{v \cdot l} l \cdot \partial k(\lambda v)\right) \mathrm{d} \mu(v) .
$$

Differentiating this formula with respect to $l^{a}$ and $s$ and using similar technique as above, one proves the remaining bounds in (67) for $|s| \geq 1$. For $|s| \leq 2$ Formula (91) should be used instead. We omit the details. 


\section{F. String-Localized Potential}

We prove here Formula (77) with (78). First, we note the following simple limiting formula. Let $f$ and $g$ be $C^{0}$-functions on $\mathbb{R}, C^{1}$ in a neighborhood of 0 , such that $f$ is bounded, and $g(u) / u$ is absolutely integrable on $|u| \geq 1$. Then

$$
\lim _{\Lambda \rightarrow \infty} \int_{\mathbb{R}} \frac{f(\tau) g(\Lambda \tau)}{\tau} \mathrm{d} \tau=\lim _{\varepsilon \searrow 0} \int_{\mathbb{R}} \frac{f(\varepsilon u) g(u)}{u} \mathrm{~d} u=f(0) \int_{\mathbb{R}} \frac{g(u)}{u} \mathrm{~d} u,
$$

all integrals in the principal value sense. The first equality is by the change of integration variable $\tau=\varepsilon u, \varepsilon \Lambda=1$. The second formula is obviously true for integration restricted to $|u| \geq 1$. For $|u| \leq 1$ the principal value integral in the middle may be written as

$$
f(0) \int_{-1}^{1} \frac{g(u)-g(0)}{u} \mathrm{~d} u+\varepsilon \int_{-1}^{1} \frac{f(\varepsilon u)-f(0)}{\varepsilon u} g(u) \mathrm{d} u .
$$

The first term gives the value on the rhs of (93), and the second term vanishes in the limit.

Next, we note that formula (76) may be written as

$$
A_{b}^{\mathrm{sl}}(x, h)=A_{b}(x)+\int\left\{\int_{\mathbb{R}_{+}} e^{a} \partial_{b} A_{a}(x+\lambda e) h(e) \mathrm{d} \lambda\right\} \mathrm{d} \nu(e),
$$

where for simplicity let $h(e)$ be compactly supported. For $e$ in a compact subset of the hyperboloid one has $\left|\partial_{b} A_{a}(x+\lambda e)\right| \leq$ const $/(1+\lambda)^{2}$ (the constant depending on $x$ and the support in $e$, use e.g., Theorem 18 in [15]), so the integrand of the iterated integral above is absolutely integrable, and we can change the order of integration. Let us denote

$$
k^{a}(\tau, l)=\int e^{a} h(e) \delta(\tau-e \cdot l) \mathrm{d} \nu(e),
$$

which is a smooth function of compact support in $\tau / l^{0}$. Then, using (4) we find

$$
\int e^{a} \partial_{b} A_{a}(x+\lambda e) h(e) \mathrm{d} \nu(e)=-\frac{1}{2 \pi} \int l_{b} k(\tau, l) \cdot \ddot{V}(x \cdot l+\lambda \tau, l) \mathrm{d} \tau \mathrm{d}^{2} l .
$$

Integrating this with respect to $\lambda$ on $[0, \Lambda]$, we get

$$
-\frac{1}{2 \pi} \int l_{b} k(\tau, l) \cdot \frac{\dot{V}(x \cdot l+\Lambda \tau, l)-\dot{V}(x \cdot l, l)}{\tau} \mathrm{d} \tau \mathrm{d}^{2} l .
$$

This may be separated into the difference of two integrals, with $1 / \tau$ treated in the principal value sense. Taking the limit $\Lambda \rightarrow \infty$ with the use of formula (93), setting (94) and integrating with respect to $\tau$, we obtain

$$
\frac{1}{2 \pi} \int l_{b}\left\{\int e^{a} h(e) \delta(e \cdot l) \mathrm{d} \nu(e) \int_{\mathbb{R}} \frac{\dot{V}_{a}(\tau, l)}{x \cdot l-\tau} \mathrm{d} \tau+\int \frac{e^{a} h(e)}{e \cdot l} \mathrm{~d} \nu(e) \dot{V}_{a}(x \cdot l, l)\right\} \mathrm{d}^{2} l,
$$

which reproduces the gauge terms in (77)-(78). 


\section{References}

[1] Ashtekar, A.: Asymptotic Quantization. Bibliopolis, Naples (1986)

[2] Brunetti, R., Guido, D., Longo, R.: Modular localization and Wigner particles. Rev. Math. Phys. 14, 759-786 (2002)

[3] Bramson, B.D.: Physics in cone space. In: Esposito, F.P., Witten, L. (eds.) Asymptotic Structure of Space-Time, pp. 273-359. Plenum, New York (1977)

[4] Bratteli, O., Robinson, D.W.: Operator Algebras and Quantum Statistical Mechanics 2, 2nd edn. Springer, New York (1996)

[5] Buchholz, D.: On the structure of local quantum fields with non-trivial interaction. In: Proceedings of the International Conference on Operator Algebras, Ideals and Their Applications in Theoretical Physics, Leipzig 1977. Teubner, Leipzig (1978)

[6] Buchholz, D., Ciolli, F., Ruzzi, G., Vasselli, E.: The universal C*-algebra of the electromagnetic field. Lett. Math. Phys. 106, 269-285 (2016). https://doi.org/ 10.1007/s11005-015-0801-y

[7] Dirac, P.A.M.: Gauge-invariant formulation of quantum electrodynamics. Can. J. Phys. 33, 650-660 (1955)

[8] Duch, P.: Infrared problem in perturbative quantum field theory. Rev. Math. Phys. 33, 2150032 (2021). arxiv.org/abs/1906.00940

[9] Fock, V.A.: Die Eigenzeit in der klassischen und in der Quantenmechanik. Phys. Z. Sowjetunion 12, 404-425 (1937)

[10] Gracia-Bondía, J.M., Mund, J., Várilly, J.C.: The chirality theorem. Ann. Henri Poincaré 19, 843-874 (2018)

[11] Herdegen, A.: Long-range effects in asymptotic fields and angular momentum of classical field electrodynamics. J. Math. Phys. 36, 4044-4086 (1995)

[12] Herdegen, A.: Semidirect product of CCR and CAR algebras and asymptotic states in quantum electrodynamics. J. Math. Phys. 39, 1788-1817 (1998). arxiv.org/abs/hep-th/9711066

[13] Herdegen, A.: Infrared problem and spatially local observables in electrodynamics. Ann. Henri Poincaré 9, 373-401 (2008). https://doi.org/10.1007/ s00023-008-0359-7

[14] Herdegen, A.: Asymptotic structure of electrodynamics revisited. Lett. Math. Phys. 107, 1439-1470 (2017). https://doi.org/10.1007/s11005-017-0948-9

[15] Herdegen, A.: Infrared problem vs gauge choice: scattering of classical Dirac field. Ann. Henri Poincaré 22, 2227-2282 (2021). https://doi.org/10.1007/ s00023-020-01015-y

[16] Jackson, J.D.: From Lorenz to Coulomb and other explicit gauge transformations. Am. J. Phys. 70, 917-928 (2002)

[17] Jauch, J.M., Rohrlich, F.: The Theory of Photons and Electrons, 2nd edn. Springer, Berlin (1976)

[18] Lee, J.M.: Introduction to Smooth Manifolds, 2nd edn. Springer, New York (2013)

[19] Leupold, S.: Feynman rules in radial gauge. arXiv:hep-th/9609222 (1996)

[20] Mund, J., Rehren, K.-H., Schroer, B.: Gauss' law and string-localized quantum field theory. JHEP 01, 001 (2020) 
[21] Mund, J., Schroer, B., Yngvason, J.: String-localized quantum fields and modular localization. Commun. Math. Phys. 268, 621-672 (2006)

[22] Reed, M., Simon, B.: Methods of Modern Mathematical Physics, vol. 3. Academic Press, San Diego (1979)

[23] Roepstorff, G.: Coherent photon states and spectral condition. Commun. Math. Phys. 19, 301-314 (1970)

[24] Schwinger, J.: On gauge invariance and vacuum polarization. Phys. Rev. 82, 664-679 (1951)

[25] Steinmann, O.: Perturbative Quantum Electrodynamics and Axiomatic Field Theory. Springer, Berlin (2000)

\section{Andrzej Herdegen}

Institute of Theoretical Physics

Jagiellonian University

ul. S. Łojasiewicza 11

30-348 Kraków

Poland

e-mail: herdegen@th.if.uj.edu.pl

Communicated by Karl-Henning Rehren.

Received: July 5, 2021.

Accepted: January 5, 2022. 